\title{
An illumination effect and an eccentric orbit for the symbiotic binary PU Vul revealed by 32 years of optical spectroscopy
}

\author{
Virginia A. Cúneo, ${ }^{1,4 \star}$ Scott J. Kenyon, ${ }^{2}$ Mercedes N. Gómez, ${ }^{3,4}$ Drahomir Chochol, ${ }^{5}$ \\ Sergey Y. Shugarov ${ }^{5,6}$ and Eugeni A. Kolotilov ${ }^{6}$ \\ ${ }^{1}$ Instituto Argentino de Radioastronomía (CCT La Plata, CONICET), C.C.5, (1984) Villa Elisa, Buenos Aires, Argentina \\ ${ }^{2}$ Smithsonian Astrophysical Observatory, 60 Garden Street, Cambridge, MA 02138, USA \\ ${ }^{3}$ Universidad Nacional de Córdoba, Observatorio Astronómico, Laprida 854, X5000BGR, Córdoba, Argentina \\ ${ }^{4}$ Consejo Nacional de Investigaciones Científicas y Técnicas (CONICET), Argentina \\ ${ }^{5}$ Astronomical Institute, Slovak Academy of Science, 05960 Tatranská Lomnica, Slovakia \\ ${ }^{6}$ Sternberg Astronomical Institute, University avenue 13, Moscow 119992, Russia
}

Accepted XXX. Received YYY; in original form ZZZ

\begin{abstract}
We analyze $\sim 32$ years of optical spectra and photometry for the symbiotic binary PU Vul. Light curves for the He I $\lambda 4471$, He II $\lambda 4686$ and $\mathrm{H} \beta \lambda 4861$ emission lines reveal an illumination effect, where the hot white dwarf ionizes the outflowing wind of the red giant, and evidence for an eccentric orbit with $\mathrm{e} \geq 0.16$. Along with the gradual appearance of high ionization emission from [Fe VII] and $\mathrm{O}$ VI, the relative fluxes of these lines suggest an increase in the effective temperature of the hot component, from roughly $10^{5} \mathrm{~K}$ on JD 2448000 (1990) to roughly $2 \times 10^{5} \mathrm{~K}$ on JD 2455000 (2009). During this period, the luminosity of the hot component dropped by a factor of 4-6 to a current value of roughly $1000 \mathrm{~L}_{\odot}$.
\end{abstract}

Key words: binaries: spectroscopic - binaries: symbiotic - stars: individual: PU Vul

\section{INTRODUCTION}

Symbiotic stars are interacting binary systems where a main sequence, subdwarf, or white dwarf (WD) star accretes material from the wind of an evolved late-type star (e.g. Aller 1984; Kenyon 1986a). Based on the amplitude $(\Delta V)$ and duration $(\Delta t)$ of their outbursts at optical wavelengths, these systems are divided into a group of classical symbiotics $(\Delta V \lesssim 2-3 \mathrm{mag}, \Delta t \lesssim 1-2$ years $)$ and symbiotic novae ( $\Delta V \approx 3-6 \mathrm{mag}, \Delta t \gtrsim 10-100$ years). Among the symbiotic novae, several (AG Peg, RT Ser, RR Tel, and PU Vul) transform into A-F supergiants at optical maximum, gradually evolve towards hotter temperatures, and then develop strong emission lines similar to a planetary nebula (e.g., Allen 1980; Iben 1982; Kenyon \& Truran 1983; Mikolajewska \& Kenyon 1992; Iben \& Tutukov 1996). In others (V1016 Cyg, V1329 Cyg, and HM Sge), the lifetime as an A-F supergiant is negligible; the strong nebular emission line spectrum appears either simultaneously with or almost immediately after optical maximum (Murset \& Nussbaumer 1994).

PU Vul was discovered by Y. Kuwano (Kozai et al. 1979) and M. Honda (Argyle et al. 1979). Before its slow

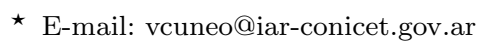

rise in 1978-1979 (JD 2443660-2443969), the system varied between $\mathrm{B}=14.5$ and $\mathrm{B}=16.6$ (Liller \& Liller 1979). At optical maximum, $\mathrm{PU} \mathrm{Vul}$ reached $\mathrm{V} \approx 9$. In 1980 (JD 2444532), the system fell almost 5 magnitudes in $\mathrm{V}$, maintained this level for more than a year, and then recovered to $\mathrm{V} \approx 9$. After $7-8$ years, the system began a slow optical decline interrupted by a second shallower minimum in 1994 (JD 2449447) and a third very shallow minimum observed only in the U-band in 2007 (JD 2454334, Shugarov et al. 2012). As the luminosity declined, the radius of the WD shrunk from $\sim 0.35 \mathrm{R}_{\odot}$ to $\sim 0.04 \mathrm{R}_{\odot}$. Shortly after the second minimum, the system became faint enough to reveal pulsations in the red giant (RG) primary with a period of $\sim 218$ days (Chochol et al. 1998; Kato et al. 2012; Shugarov et al. 2012).

Throughout these epochs, optical and ultraviolet (UV) spectroscopic data provided windows into the evolution of the eruptive star. Shortly after outburst, the optical spectrum resembled an F-type supergiant (Yamashita et al. 1982; Kanamitsu 1991). Although this component disappeared during the 1980 minimum, it returned during the recovery to the second optical maximum. From 1982-1990, the spectrum evolved from an F-type star into an A-type star with occasional H I Balmer emission lines, then to a mid- 
dle B-type star with strong Balmer and weak Fe II emission lines, and finally to a very blue star with broad He II lines characteristic of Wolf-Rayet (WR) stars (Belyakina et al. 1989; Kenyon 1986b; Iijima 1989; Kanamitsu et al. 1991; Gochermann 1991; Tomov et al. 1991; Vogel \& Nussbaumer 1992; Sion et al. 1993; Klein et al. 1994). During this transformation, red optical spectra showed prominent $\mathrm{TiO}$ absorption bands characteristic of M-type giant stars. Together with spectra acquired during the 1994 minimum, these data demonstrated that the system is a binary composed of a pulsating M6 giant and an eruptive WD. During the deep minima, the RG occults the WD and any surrounding ionized material. Thus, the three minima provide a rough estimate of the orbital period, 13.4 years (Friedjung et al. 1984; Kenyon 1986b; Nussbaumer \& Vogel 1996; Garnavich 1996; Tatarnikova et al. 2011; Kato et al. 2012; Shugarov et al. 2012, and references therein).

In current theory, symbiotic nova eruptions result from thermonuclear runaways in accreted material on the surface of the WD (e.g. Starrfield et al. 1972; Kato 2002; Yaron et al. 2005). During the decline from optical maxima, the WD should evolve in effective temperature from 5,000-6,000 K to $150,000-200,000 \mathrm{~K}$ at roughly constant luminosity and then evolve to lower luminosity at roughly constant radius. For PU Vul, detailed analyses of UV data appear to support this prediction (Kato et al. 2012): to within a factor of two, the hot WD maintained a constant luminosity from 1982-2011 as its effective temperature increased from $6,500 \mathrm{~K}$ to $150,000-165,000 \mathrm{~K}$. However, optical spectra suggest a dramatic decline in luminosity, from $\sim 10^{4} \mathrm{~L}_{\odot}$ to $\sim 10^{3} \mathrm{~L}_{\odot}$ over 1992-2008 (Tatarnikova \& Tatarnikov 2009; Tatarnikova et al. 2011; Tatarnikova et al. 2018). Contrary to theoretical predictions, these data also imply an increase in effective temperature as the bolometric luminosity declined.

To try to resolve this difference, we analyze optical spectrophotometry of PU Vul acquired from 1984-2016 (JD 2445804-2457724). Aside from adding to our understanding of the evolution from an F-type supergiant into a WR-type star (see also Kenyon 1986b), these data probe the evolution of emission lines during epochs when the luminosity remained roughly constant (Kato et al. 2012) or declined by a factor of 10 (Tatarnikova et al. 2011). The dense timecoverage of our spectra allows us to probe the evolution of the hot component on short and long time scales.

After summarizing the observations in $\S 2$, we describe the general evolution of the spectrum, the evolution of fluxes for selected $\mathrm{H}$ and $\mathrm{He}$ emission lines, and a comparison with previous observations and theoretical predictions in $\S 3$. We then discuss the illumination effect, a new estimate of the orbital eccentricity, and the evolution of the temperature and luminosity of the hot component in $\S 4$. We conclude with a discussion of the main results in $\S 5$.

\section{OBSERVATIONS}

\subsection{Optical Spectroscopy}

Throughout 1984-92 (JD 2445804-2448935), S. K. acquired occasional low resolution optical spectra of PU Vul with the cooled dual-beam intensified Reticon scanner (IRS) or the GoldCam CCD dewar mounted on the white spectrograph of the KPNO No. 1 0.9-m telescope. Observations of 5-10 standard stars place the spectra on the Hayes \& Latham (1975) flux scale. Typical errors in the flux calibration are $3 \%$ to $5 \%$. For the IRS, blue (3500-6200 А) and red (5800-8400 А) spectra acquired on consecutive nights yield an independent measure of the accuracy of the flux calibration. For the region of overlap, the difference in the continuum level is small, $\lesssim 3 \%$. For these 21 spectra, the typical signal-to-noise in the continuum at $5500 \mathrm{~A}$ is $15-20$ per pixel.

From 1994-2016 (JD 2449694-2457724), P. Berlind, M. Calkins, and several other observers acquired low resolution optical (3500-7300 ̊) spectra of PU Vul with FAST, a high throughput, slit spectrograph mounted at the Fred L. Whipple Observatory 1.5-m telescope on Mount Hopkins, Arizona (Fabricant et al. 1998). They used a $300 \mathrm{~g} \mathrm{~mm}^{-1}$ grating blazed at $4750 \AA$, a $3^{\prime \prime}$ slit, and a thinned $512 \times 2688 \mathrm{CCD}$. These spectra cover $3800-7500 \AA$ at a resolution of $6 \AA$. We wavelength-calibrate the spectra in NOAO IRAF ${ }^{1}$. After trimming the CCD frames at each end of the slit, we correct for the bias level, flat-field each frame, apply an illumination correction, and derive a full wavelength solution from calibration lamps acquired immediately after each exposure. The wavelength solution for each frame has a probable error of $\pm 0.5 \AA$ or better. To construct final 1-D spectra, we extract object and sky spectra using the optimal extraction algorithm APEXTRACT within IRAF.

We acquired 458 FAST spectra with exposure times of $1-300$ seconds. To estimate the signal-to-noise $(\mathrm{S} / \mathrm{N})$ in the continuum, we examined three different regions relatively free of emission lines (4750-4840 $\mathrm{\AA}, 5900-6070 \AA$, and 6700-6800 $\AA$ ), measured fluctuations relative to the continuum level, and averaged the values for each spectrum. Most spectra have moderate signal-to-noise, $\mathrm{S} / \mathrm{N} \gtrsim 15$ per pixel, for an exposure time of 100 seconds.

To flux-calibrate the PU Vul spectra on reasonably clear nights, we observed several standards from the IRS standard star manual (Barnes \& Hayes 1982). For each spectrum, we derive the extinction as a function of wavelength using the KPNO extinction curve and a polynomial interpolation routine from Press et al. (1992). After applying the extinction correction, we bin the counts in $50 \AA$ bins centered on the wavelengths in the NOAO standard star files included with IRAF releases (e.g., the "irscal" directory). Comparison with standard star magnitudes yields the correction factor for each bin. We use a polynomial interpolation routine (Press et al. 1992) to apply the binned correction factors to the original standard star spectrum. The resulting spectrum is then calibrated on the Hayes \& Latham (1975) flux scale (see also Massey et al. 1988). As a test of the flux-calibration, we integrate the corrected spectra over $\mathrm{B}$ and $\mathrm{V}$ filter response curves; our approach yields the correct $\mathrm{B}$ and $\mathrm{V}$ magnitudes for each standard to $\pm 0.01 \mathrm{mag}$.

On any night, most standards are observed at airmasses within $\pm 0.1-0.2$ of each PU Vul spectrum. To apply the flux-calibration to PU Vul spectra, we derive the median calibration for the set of standard stars. The inter-quartile

1 IRAF is distributed by the National Optical Astronomy Observatory, which is operated by the Association of Universities for Research in Astronomy, Inc. under contract to the National Science Foundation. 
Table 1. Log of observations. We include here only the information for the first ten nights in our sample. The full version of the table is available as online-only material.

\begin{tabular}{cccc}
\hline JD & N & Etime & Cond. \\
\hline 2445804 & 1 & 480 & good \\
2445985 & 1 & 600 & good \\
2446222 & 1 & 480 & good \\
2446723 & 1 & 1200 & good \\
2447108 & 1 & 720 & good \\
2447496 & 1 & 720 & good \\
2448408 & 1 & 920 & good \\
2448526 & 1 & 960 & good \\
2448935 & 4 & 150 & good \\
2449694 & 2 & $5-60$ & good \\
\hline
\end{tabular}

range then provides an estimate of the error in the calibration. For nights with at least 4 standard star observations, the average and median calibrations agree to $\pm 0.02 \mathrm{mag}$. When nights are reasonably close to photometric, the interquartile range of the calibration is $0.02-0.03$ mag. On nonphotometric nights, the inter-quartile range is $0.1-1.5 \mathrm{mag}$. Table 1 lists the observing log; $\mathrm{N}$ is the number of individual exposures with a range of exposure times Etime. Sky conditions (Cond.) describe sky transparency as "good" when the error in the standard star observation is $<0.1 \mathrm{mag}$, "fair" when error $=0.1-0.3 \mathrm{mag}$, and "poor" for error $>0.3 \mathrm{mag}$. The calibration is good for 81 nights, fair for 52 and poor for 22 . We see that at least half of the observations need a better calibration, we decided then to use photometry from Shugarov et al. (2012) and new data acquired by the authors to minimize the systematic error, as explained in Section 3.2.

\subsection{Optical Photometry}

To improve the calibration of the FAST spectra, we compare B and V magnitudes from our FAST calibration with an extensive set of $\mathrm{B}$ and $\mathrm{V}$ photometry. As described in Shugarov et al. (2012), contemporaneous broadband UBVRI photoelectric and CCD data acquired at three different observatories were transformed to a common photometric system, $\mathrm{UBVR}_{J} \mathrm{I}_{J}$. The photometric data in Shugarov et al. (2012) cover the period 1979-2011. For FAST spectra acquired after 2011, we make a comparison with new photometry from the same observatories using the same acquisition and reduction procedures described in Shugarov et al. (2012, and references therein). The new photometry is included at the Table "new_photometry.txt", available as online-only material. Overall, these data have a typical uncertainty of 0.01-0.02 mag.

\section{SPECTROSCOPIC EVOLUTION OF PU VUL: 1984-2016}

\subsection{General Evolution of the Optical Spectrum}

To develop a better understanding of the changing optical spectrum, we first describe the long-term behavior of absorption and emission features. During 1984-92 (JD 2445804 -2448935), the optical spectrum of PU Vul evolved dramatically on the IRS spectra (Figure 1). Prior to 1987
(JD 2446723), the system resembled an A-F supergiant with occasional $\mathrm{H} \alpha$ and $\mathrm{H} \beta$ emission lines (see also Kenyon 1986b; Ivison et al. 1991). As the A-F absorption features vanished in 1987-88 (JD 2447496), the blue continuum strengthened; prominent He I and Fe II/[Fe II] emission lines also appeared (see also Iijima 1989; Gochermann 1991; Kanamitsu et al. 1991; Klein et al. 1994). In 1991-1992 (JD 2448408-2448935), the He I lines reached a maximum intensity, $\sim 2-3$ times stronger than a much broader He II $\lambda 4686$ feature. As the He I lines intensified, the standard [O III] emission lines at $\lambda 4363,4959,5007$ also strengthened, with $\lambda 4363$ ( $\lambda 5007)$ at roughly $75 \%(50 \%)$ of the intensity of $\mathrm{H} \gamma(\mathrm{H} \beta)$. In September 1991 (JD 2448526), the [N II] $\lambda 5755$ nebular line peaked at nearly $80 \%$ of He I $\lambda 5876$.

To summarize the evolution in 1994-2016 (JD 24496942457724), we rely on the FAST spectra (Figure 2). During 1994-1996 (JD 2449609-2450258), [O III] emission surpassed $\mathrm{H} \beta$ and $\mathrm{H} \gamma$. By June 2000 (JD 2451701), however, $\mathrm{H} \beta$ and $\mathrm{H} \gamma$ were much stronger than neighboring [O III] lines. As [O III] emission weakened, He II $\lambda 4686$ strengthened; relative to $\mathrm{H} \beta$, the He II $\lambda 4686$ flux grew by more than a factor of 10 by 2005 (JD 2453525). In addition to the growth in He II $\lambda 4686$ emission, the June 2005 (JD 2453525) spectrum was much richer than previous spectra, with strong emission from [Fe VII] $\lambda 3758$ and $\lambda 6087$ and the Raman scattered O VI band at 16830 . Although the emission line fluxes continue to evolve after 2005, the high excitation features remained prominent on all of the FAST spectra (see also Tatarnikova et al. 2018).

To focus on the development of WR features in 19912000 (JD 2448526-2451903), Figure 3 shows the evolution of He II $\lambda 4686$ and the N III/C III blend near $\lambda 4640$. Early on, these features were weak but broad, suggestive of outflow with a velocity of approximately $1000 \mathrm{~km} \mathrm{~s}^{-1}$ (see also Tomov et al. 1991). As these broad lines maintained a roughly constant equivalent width from 1991-1995 (JD 24485262450019), narrow emission features grew stronger. Although the broad features remained visible until 2001 (JD 2452175), they were much weaker relative to the narrow components. We do not have spectra between 2001 (JD 2452175) and 2005 (JD 2453525), but by 2005 the broad features were below our detection limit and remained undetectable through 2016 (JD 2457724).

Other WR-type emission lines - e.g., faint N IV $\lambda 4058$ (Tomov et al. 1991) - were also weakly present from May 1991 (JD 2448408) until November 1992 (JD 2448935) but were not detected during 1994-2016 (JD 2449694-2457724). Because our spectra have fairly low resolution, it is possible that these features were present but masked by numerous narrow features.

Aside from Tomov et al. (1991), Andrillat \& Houziaux (1995) reported the evolution of WR features on spectra acquired during 1991-1995 (see also Kolotilov et al. 1995). All of the broad emission lines disappeared during the 1994 minimum and re-appeared on post-eclipse spectra. Although we do not have spectra covering the minimum, He II $\lambda 4542$ and C IV emission at $\lambda 5801$ and $\lambda 7226$ were visible after the eclipse. Broad C IV $\lambda 7226$ emission disappears during 19992000 (JD 2451335-2451903); 15801 disappeared definitely by 2005 (JD 2453525). After the eclipse, Andrillat \& Houziaux (1995) also note the development of a narrow emission feature superposed on the broader WR components. They 

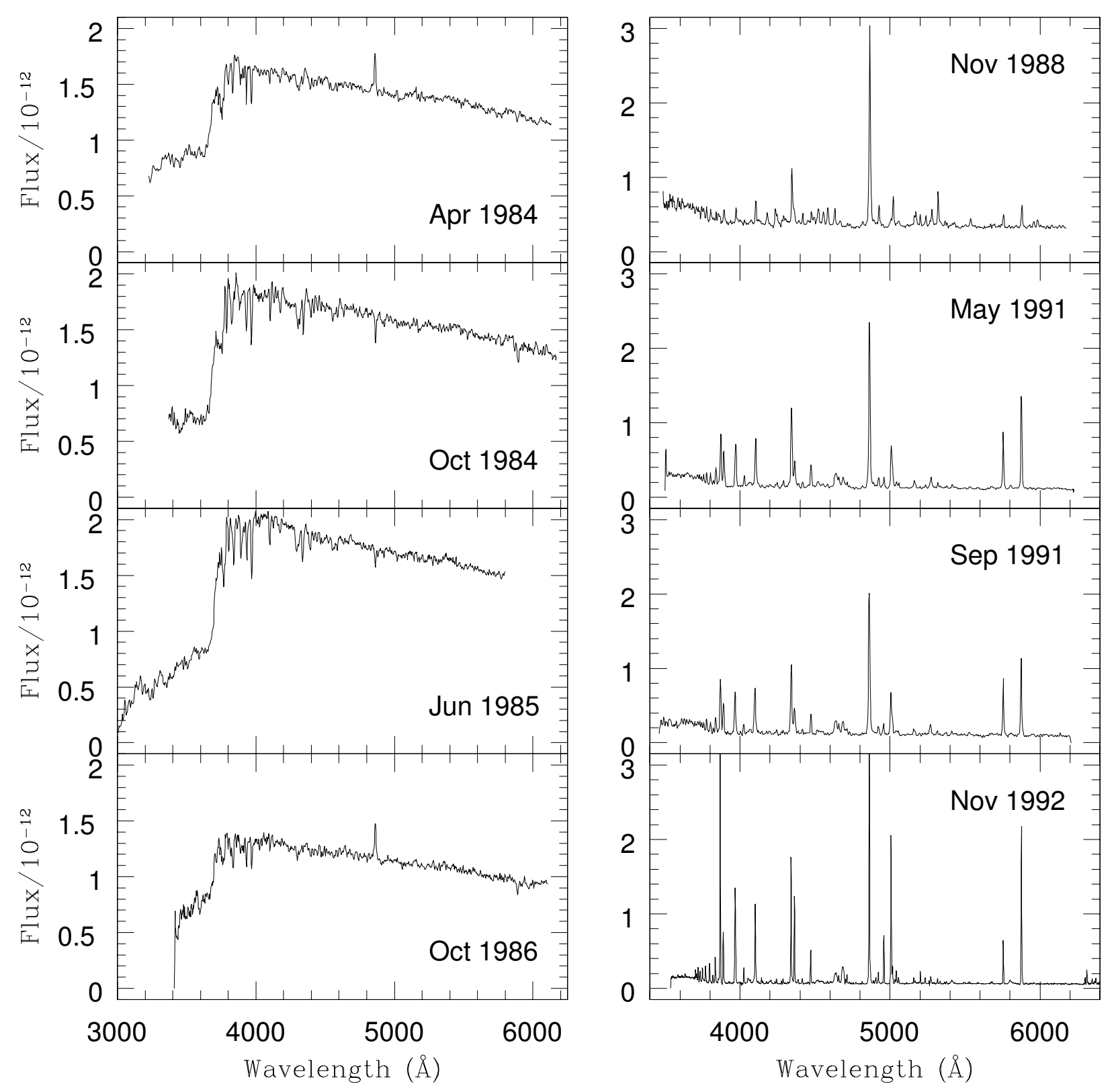

Figure 1. Sample of the PU Vul blue spectra obtained between 1984 (JD 2445804) and 1992 (JD 2448935). Fluxes are in units of erg $\mathrm{s}^{-1} \mathrm{~cm}^{-2} \AA^{-1}$.

measured a displacement of $150 \mathrm{~km} \mathrm{~s}^{-1}$ between the centroids of the narrow and broad features. On our low resolution spectra, it is impossible to measure any shift between the two components.

\subsection{Line fluxes and the light curve}

To derive equivalent widths and line fluxes, we used the splot task within IRAF. After we choose by eye two points on either side of the lines for tracing the continuum, the routine fits a gaussian profile to the line intensity. We also used the sbands task, specifying the central wavelength of the line, two well-spaced points to define the continuum, and an adequate band width to measure the line flux. Comparing the values obtained with both methods, the differences are within the measurement errors. The choice of the continuum is the main source of error. In the following discussion, we adopt fluxes measured with splot, which provides more flexibility in defining the continuum.

On most nights, we have a set of three FAST spectra with different exposure times. Short exposures avoid saturating the strongest lines but have poor $\mathrm{S} / \mathrm{N}$ for the rest of the spectrum. Intermediate and long exposures yield optimal $\mathrm{S} / \mathrm{N}$ for moderate and weak lines. For each line in this 


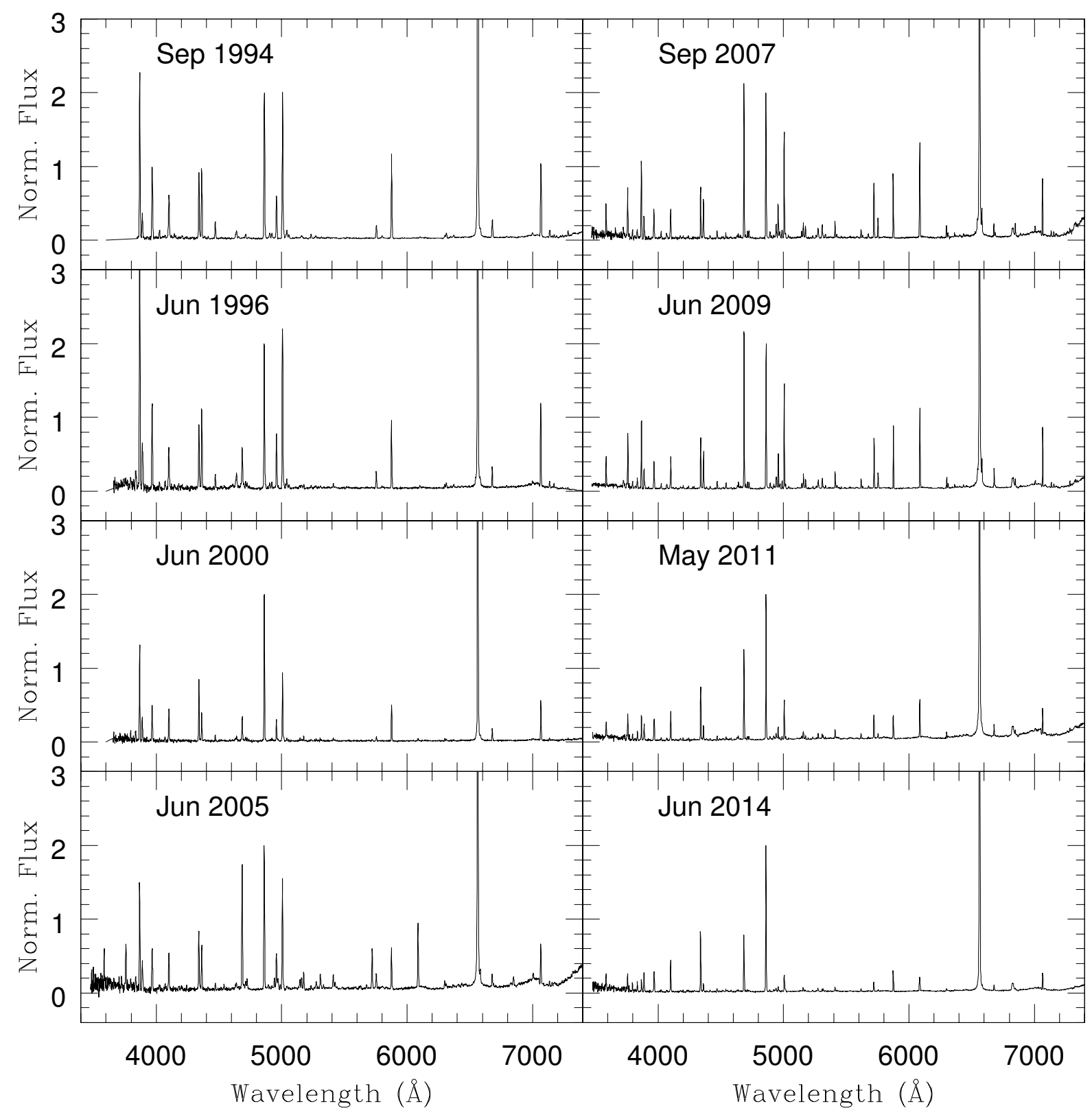

Figure 2. Sample of the PU Vul spectra obtained between 1994 (JD 2449609) and 2014 (JD 2456833). Normalized fluxes are fluxes relative to $\mathrm{H} \beta$, normalized to a value of 2 .

study, we adopted equivalent widths and fluxes from spectra with the longest exposure time which did not saturate the feature. Thus, we have one optimal measurement per feature, per night. As mentioned previously, roughly half of the spectra were acquired on nights with significant cirrus. To place all of the spectra on the same photometric scale, we normalize the fluxes to the optical broadband photometry from Shugarov et al. (2012). We interpolated the BV photometry to the dates of our FAST observations and corrected the line fluxes for He I $\lambda 4471$, He II $\lambda 4686$, and $\mathrm{H} \beta \lambda 4861$ using the difference in B magnitudes, $\Delta B=B(F A S T)-B($ phot $)$. Tests using a correction derived from $\mathrm{B}$ and $\mathrm{V}$ photometry interpolated to the wavelengths of the three lines yielded nearly identical results. Thus, we employed the simpler correction based on interpolated $B$ photometry to place all of the FAST line fluxes on the proper photometric scale. On some nights of very poor quality, the photometric calibration failed. We discarded these data. Table 2 lists the photometric calibrated fluxes for the $\mathrm{H} \gamma$ 24341, He I 14471 , He II $\lambda 4686, \mathrm{H} \beta \lambda 4861$ and $\mathrm{H} \alpha \lambda 6563$ lines.

The line fluxes we measured have several sources of error. By using the photometry to rescale fluxes, we have (i) a small error in the photometry, $\sim 0.01-0.02 \mathrm{mag}$, (ii) another modest, $\sim 0.01-0.02 \mathrm{mag}$, error from the $\mathrm{S} / \mathrm{N}$ of the spectra 


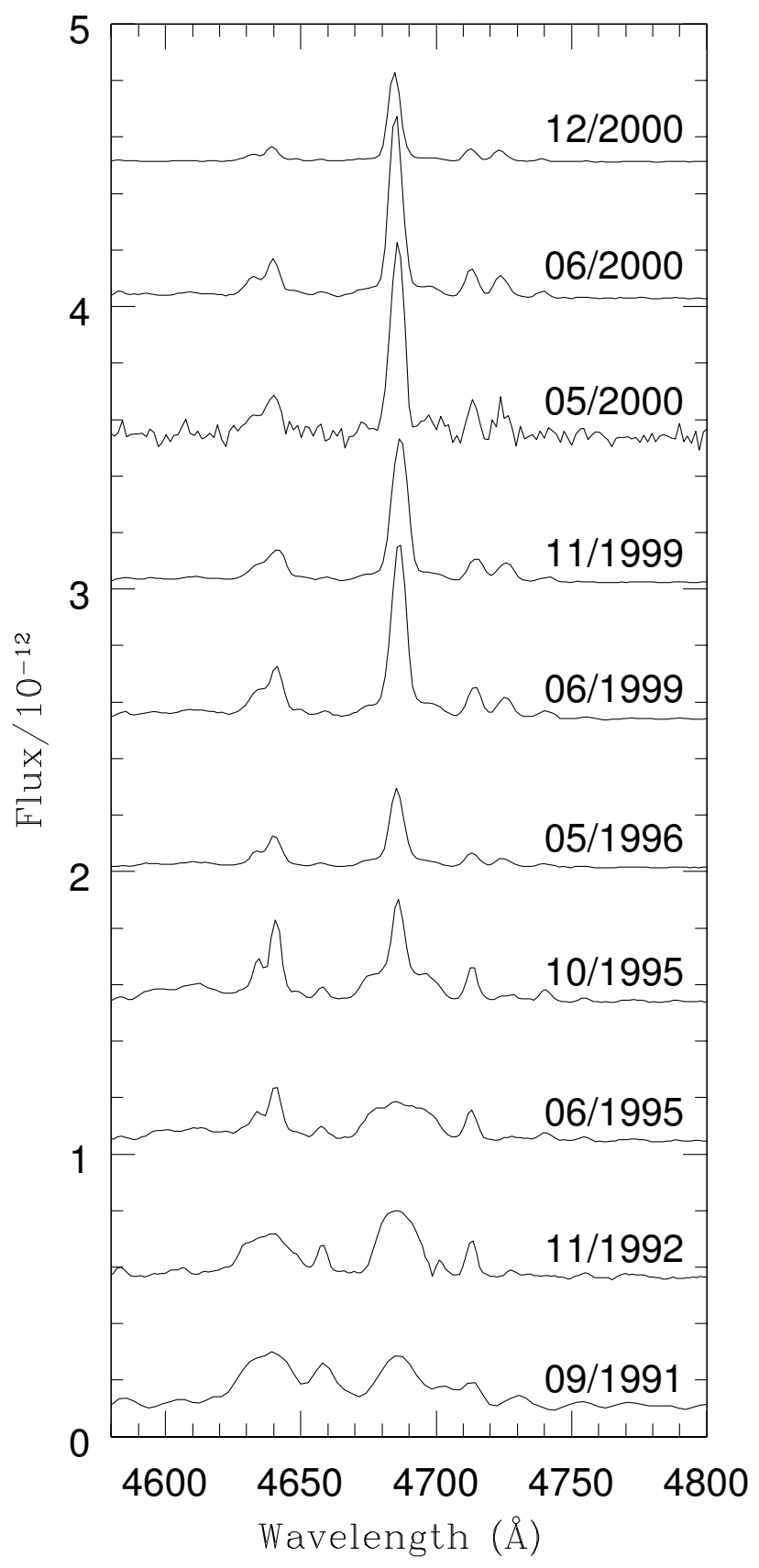

Figure 3. Evolution of He II $\lambda 4686$ between 1991 (JD 2448526) and 2000 (JD 2451903). Each spectrum was displaced a factor multiple of 0.5 from the flux base level to a better display. Fluxes are in units of erg s $\mathrm{s}^{-1} \mathrm{~cm}^{-2} \AA^{-1}$.

( 10-20) integrated over the 10 pixel width of the line, (iii) a $\sim 0.05$ mag error in the placement of continuum due to the modest $\mathrm{S} / \mathrm{N}$ of the spectra, and (iv) the error from blends in the line or any extra flux in a broad component. For these lines, blends are negligible; however, an unidentified broad component could contribute $\sim 5 \%$ to $10 \%$ of the total flux. Altogether, the errors in the fluxes are $\sim 10 \%$ to $15 \%$ with the dominant sources of uncertainty as error in the continuum fit and error in the flux from a broad component.

The top three panels of Figure 4 show the photome-
Table 2. Line fluxes in units of $10^{-12} \mathrm{erg} \mathrm{s}^{-1} \mathrm{~cm}^{-2}$. We include here only the line fluxes for the first ten spectra in our sample. The full version of the table is available as online-only material.

\begin{tabular}{cccccc}
\hline $\mathrm{JD}$ & $\mathrm{H} \gamma$ & $\mathrm{He}$ I $\lambda 4471$ & $\mathrm{He}$ II $\lambda 4686$ & $\mathrm{H} \beta$ & $\mathrm{H} \alpha$ \\
\hline 2445804 & 1.98 & 0.00 & 0.00 & 4.61 & 45.37 \\
2445985 & 1.08 & 0.03 & 0.00 & 2.70 & 26.10 \\
2446222 & 0.58 & 0.00 & 1.04 & 1.52 & 16.58 \\
2446723 & 1.67 & 0.00 & 0.00 & 4.63 & 48.90 \\
2447108 & 7.34 & 0.05 & 0.00 & 19.83 & 202.51 \\
2447496 & 12.25 & 0.08 & 0.00 & 29.88 & 294.75 \\
2448408 & 8.27 & 0.14 & 1.44 & 23.63 & 227.60 \\
2448526 & 9.35 & 0.12 & 2.30 & 22.00 & 210.21 \\
2448935 & 8.46 & 0.14 & 4.51 & 22.27 & 205.45 \\
2449694 & 1.66 & 0.62 & 0.79 & 4.04 & 39.55 \\
\hline
\end{tabular}

try corrected fluxes for the He I $\lambda 4471$, He II $\lambda 4686$ and $\mathrm{H} \beta \lambda 4861$ lines as a function of orbital phase and time for the period between April 1984 (JD 2445804) and December 2016 (JD 2457724). The orbital phase was derived from the ephemeris $\mathrm{JD}_{\text {Min }}=2444537+4901 \times E$, a linear fit to the three determined primary minima at JD 2444532, 2449447 and 2454334 (Kato et al. 2012; Shugarov et al. 2012).

The bottom panel of Figure 4 shows the light curve of PU Vul in the U, B, V, R and I bands, for the same time period. Within these light curves, the midpoints for eclipses 2 (1994) and 3 (2007) are denoted with green arrows. Aside from the eclipses and the abrupt decline starting around phase 0.5 , there are several clear features in the light curve: (i) a sinusoidal oscillation in the brightness with maxima denoted by purple arrows (and slightly to the right for the R-band) and (ii) variations generated by the pulsation of the $\mathrm{RG}$, which are more prominent after the eclipse at phase 2.0 (see, for example, Shugarov et al. 2012).

Despite the lack of spectra before 1994 (JD 2449694), the rise in $\mathrm{H} \beta$ and He I fluxes is clearly associated with the drop in optical brightness starting at phase 0.5. The $\mathrm{H} \beta$ line seems to reach a peak sometime during phase $0.6-0.8$, while the He I $\lambda 4471$ line shows a maximum close to phase 1.1, after the eclipse. From our limited phase coverage during this epoch, there is little evidence for an eclipse in any of the three lines. After the eclipse, He I emission declines, reaching a clear minimum sometime during phase $1.75-2$. However, there is a small increase in emission that coincides with the optimal maximum near phase 2.5 .

The $\mathrm{H} \beta$ line (i) recovers from its decline to reach a second maximum which coincides with the maximum in the broadband light curve around phase 1.4, (ii) declines to a minimum around phase 2.0 , and (iii) recovers again to a third maximum when the broadband light is bright just before phase 2.5. Comparing the three maxima in $\mathrm{H} \beta$, there appears to be a decline in flux from the first maximum to the second and third maxima. However, this decline is small compared to the overall fluctuation in $\mathrm{H} \beta$ emission.

For the He II $\lambda 4686$ line, a similar behavior is observed. Due to the lack of data before the eclipse, we can not say much about the behavior of the line. But, as for $\mathrm{H} \beta$, there seems to be a maximum around phase 1.4. Then the flux declines reaching a minimum at phase 2 , which coincides with the third eclipse. And finally, the flux rises again reaching 


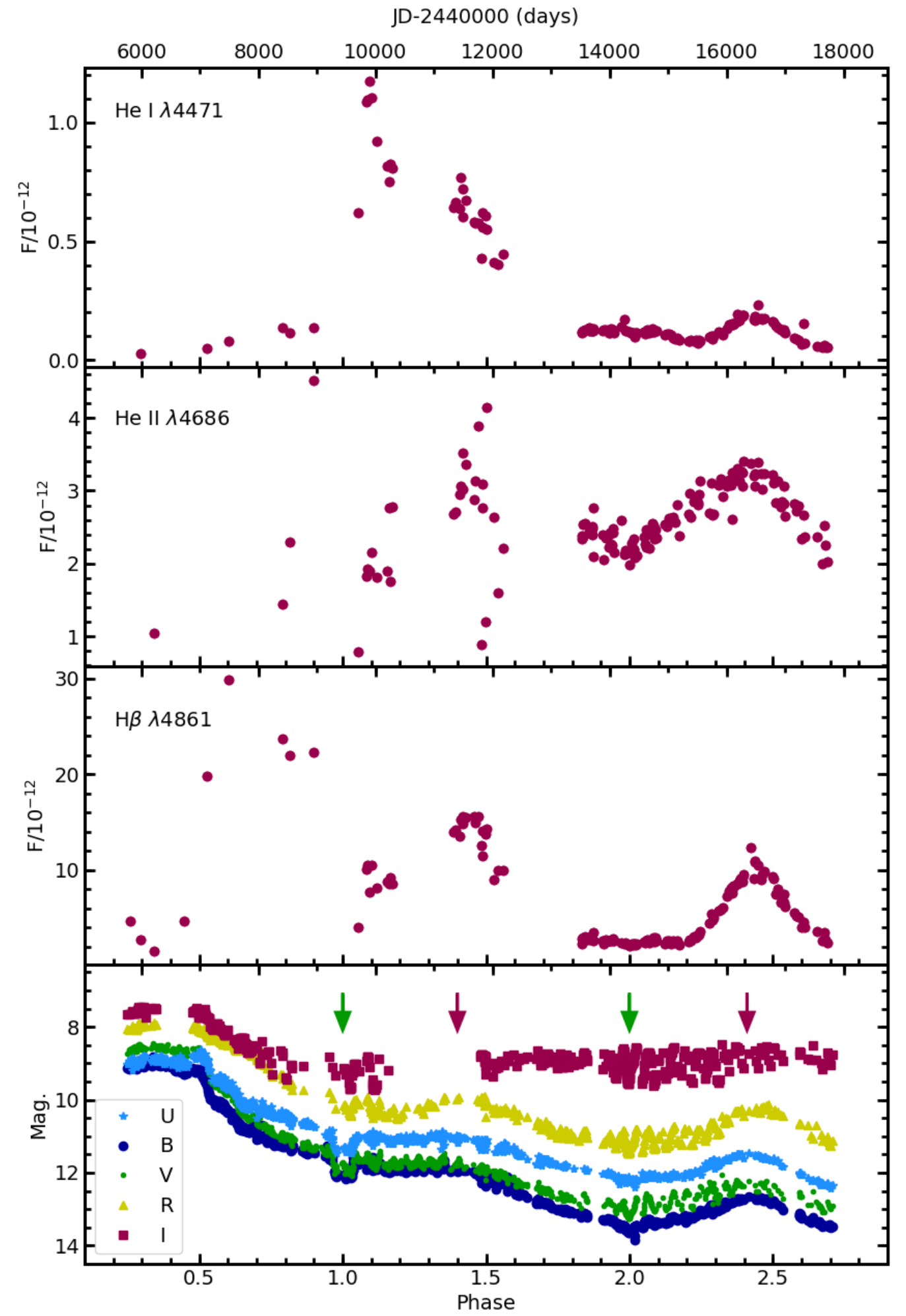

Figure 4. Fluxes as function of phase and time for He I $\lambda 4471$, He II $\lambda 4686$ and H $\beta \lambda 4861$ lines (top three panels) and the light curve (bottom panel), in the U, B, V, R and I bands, of PU Vul. Fluxes are in units of $\mathrm{erg} \mathrm{s}^{-1} \mathrm{~cm}^{-2}$. The green arrows point the second and third eclipses at phases 1 and 2, respectively; the purple arrows denote the epochs of maximum light at phases $\sim 1.4$ and $\sim 2.4$. 
Table 3. He II $\lambda 4686$ broad and narrow fluxes, normalized to the B-band photometry.

\begin{tabular}{ccc}
\hline JD & $\begin{array}{c}\mathrm{F}_{\text {Broad }} \\
{\left[\times 10^{-12} \mathrm{erg} \mathrm{s}^{-1} \mathrm{~cm}^{-2}\right]}\end{array}$ & $\begin{array}{c}\mathrm{F}_{\text {Narrow }} \\
{\left[\times 10^{-12} \mathrm{erg} \mathrm{s}^{-1} \mathrm{~cm}^{-2}\right]}\end{array}$ \\
\hline 2450019 & 1.696 & 0.124 \\
2450192 & 1.337 & 0.556 \\
2450231 & 1.048 & 1.715 \\
2450258 & 1.406 & 1.925 \\
2450284 & 1.300 & 1.474 \\
2451335 & 0.773 & 1.901 \\
2451369 & 0.978 & 2.028 \\
2451431 & 0.876 & 2.074 \\
2451454 & 0.097 & 2.972 \\
2451484 & 0.964 & 2.558 \\
2451495 & 0.819 & 2.694 \\
2451690 & 0.669 & 2.343 \\
2451701 & 0.827 & 2.309 \\
2451762 & 0.989 & 2.585 \\
2451822 & 1.119 & 1.967 \\
2451824 & 0.573 & 2.190 \\
2451903 & 0.936 & 3.206 \\
2452026 & 1.331 & 1.136 \\
2452175 & 1.741 & 0.709 \\
\hline
\end{tabular}

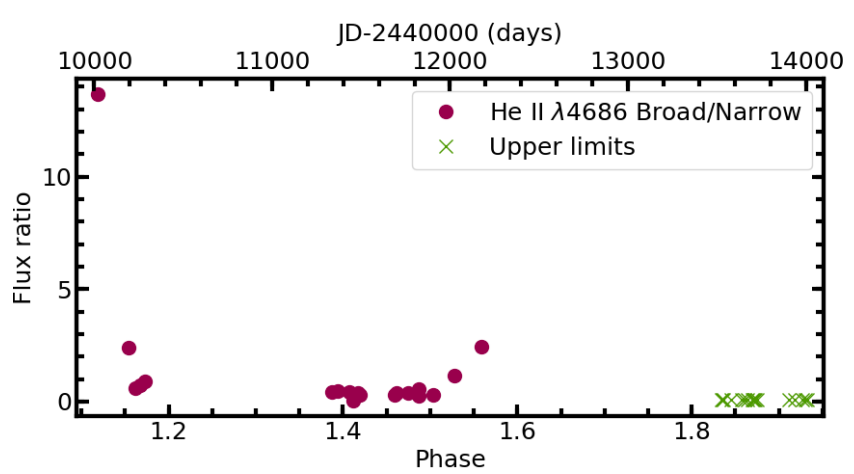

Figure 5. Broad to narrow component ratio for the He II $\lambda 4686$ line flux between JD 2450018 (October 1995) and JD 2454006 (September 2006).

a maximum before phase 2.5. Long-term fluctuations in the R-band are clearly phased with variations in the three lines. Between $\sim 1995 \quad$ (JD $\sim 2450018)$ and $\sim 2001$ (JD 2452175), He II $\lambda 4686$ is composed of a broad and a narrow component. Prior to 1995, the line is either absent or consists only of the broad component. After 2001, only the narrow component is visible. For the period with two components, we fit two gaussians to derive the relative contribution of each component to the total flux (see Table $3)$. Figure 5 shows the flux ratio of the broad to the narrow component derived from the two-gaussian fits. Aside from two spectra near JD $\sim 2452000$, the broad component gradually weakened relative to the narrow component. Once our spectroscopic observations resumed after JD $\sim 2453525$, the ratio of the broad to narrow component is always smaller than $\sim 0.1-0.2$ and sometimes close to zero. Due to the gap in our data during JD $\sim 2452175-2453525$, we cannot know when the broad component weakens after JD $\sim 2452175$.

Overall, the broad component of He II $\lambda 4686$ has a width consistent with the WR features observed in other symbiotic stars (Figure 3). Once it became visible, the narrow component of the He II $\lambda 4686$ line has a width similar to $\mathrm{H} \beta$. On all our spectra, there is no evidence for broad features on He I $\lambda 4471$ or $\mathrm{H} \beta$. Thus the broad components of these two lines have fluxes less than $10 \%$ of our measured fluxes.

\subsection{Comparison with other works}

From the tight variation of the $\mathrm{H} \beta$ and $\mathrm{He} \mathrm{I} \lambda 4471$ lines at orbital phases $1.8-2.7$, the relative calibration of our line fluxes appears excellent. The He II $\lambda 4686$ line shows more scatter in this period, but the scatter is still small relative to the amplitude of the variations. To judge the accuracy of the absolute calibration, we consider comparisons with other investigators. Although data from Tatarnikova et al. (2011) cover a much shorter time period (2001-2008, JD 2452167$2454763)$, results for nights reasonably close in time yield flux ratios $F_{T} / F_{0}=4.22 \pm 2.74$ (He I $\left.\lambda 4471\right), 1.61 \pm 0.12$ (He II 14686) and $1.71 \pm 0.18(\mathrm{H} \beta)$, where $F_{T}$ is the flux listed in Table 3 of Tatarnikova et al. (2011) and $F_{0}$ is our measurement. Despite the quoted $10 \%$ to $15 \%$ uncertainty in both sets of fluxes, our measurements are roughly $60 \%$ to $70 \%$ of theirs. Still, the flux ratios are consistent within the $3 \sigma$ uncertainties. Compared to data for He II $\lambda 4686$ in Andrillat \& Houziaux (1995), our fluxes are roughly $50 \%$ larger before the eclipse of 1994 and a factor of 2-3 smaller after the eclipse. In both cases, it is possible that significant week-toweek or month-to-month variations complicate comparisons of different data sets.

As another test, we consider the flux ratios of several $\mathrm{H}$ and He lines. For the $\mathrm{H}$ I Balmer lines, case B recombination predicts ratios of $0.45-0.5$ for $\mathrm{H} \gamma / \mathrm{H} \beta$ and $2.7-3$ for $\mathrm{H} \alpha / \mathrm{H} \beta$ (Osterbrock 1989). With $E(B-V)=0.3$, our results are $0.41 \pm 0.04$ for $\mathrm{H} \gamma / \mathrm{H} \beta$ and $7.3 \pm 0.5$ for $\mathrm{H} \alpha / \mathrm{H} \beta$. As is typical for symbiotic stars, the data suggest a modest optical depth $-\tau \approx 5-10$ in the $\mathrm{H} \alpha$ line. Our results for the ratio of He II $\lambda 5411$ ( $\lambda 4542)$ to He II $\lambda 4686,0.085 \pm 0.003$ $(0.041 \pm 0.013)$ are identical to the predicted $0.08(0.036)$. Finally, published fluxes for the He II $\lambda 1640$ lines (Tatarnikova \& Tatarnikov 2009; Kato et al. 2011) - dereddened with $E(B-V)=0.3$ (Savage \& Mathis 1979; Kato et al. 2012) - yield $F(\lambda 1640) / F(\lambda 4686)=4.44 \pm 1.23$, which is reasonably close to the predicted case B value of 6-7 (Aller 1984). Hillier (1987) noted that the optical depth in the He II lines of WR stars often yields ratios far from the case $\mathrm{B}$ value. The large variation in the ratio of He II $\lambda 1640$ to $\lambda 4686$ in PU Vul is probably also due to large optical depth.

Finally, we compare the predicted nebular continuum emission (from He II $\lambda 4686$ and $\mathrm{H} \beta$ ) with the observed continuum levels from the FAST spectra and the UBVRI photometry. From case B recombination theory, the predicted continuum level is a simple function of the line fluxes, the electron density $n_{e}$, and the electron temperature $T_{e}$ (Aller 1984; Osterbrock 1989). For $n_{e} \gtrsim 10^{6} \mathrm{~cm}^{-3}$ and $T_{e} \approx 10,000$ $20,000 \mathrm{~K}$, we derive the predicted continuum flux at $4400 \AA$ for each set of line fluxes. Over the complete set of FAST spectra, the predicted continuum fluxes are roughly $25 \%$ to close to $100 \%$ of the observed continuum level. Following the same procedure for $3500-4000 \AA$, the predicted level is again a significant fraction of the observed U-band flux. The observed line fluxes are therefore consistent with the observed 
continuum level at short wavelengths. At longer wavelengths (e.g., $\mathrm{V}$ and $\mathrm{R}$ bands), the predicted nebular continuum is much smaller. Considering the much larger contribution of the RG to the observed continuum (see below), the predicted nebular continuum is also consistent with the observations at these wavelengths.

\section{ANALYSIS}

\subsection{Illumination effect and orbital eccentricity}

For phases 1.8-2.7, the time variation of the broadband light curve and the optical line fluxes shows clear evidence for an illumination effect, where high energy photons from the hot WD illuminate the outer atmosphere and the outflowing wind of the RG by photoionizing $\mathrm{H}, \mathrm{He}$ and other elements, which then recombine to produce line and continuum emission (Seaquist et al. 1984; Nussbaumer \& Vogel 1987; Proga et al. 1996, 1998). Near phase 2, the dips in the U light curve and the He II $\lambda 4686$ flux suggest an eclipse, which is observed more clearly at phase 1 . During these epochs, the RG occults the hot WD and the surrounding nebula. Also, as the densest part of the ionized wind lies between the hot component and the RG, when the RG is in front of the WD it occults a portion of this region, so the observed flux is low. Because the WD is small and the ionized nebula is optically thin, the hot component cannot occult the RG. Roughly $40 \%$ of an orbital cycle after the eclipse of the WD, when the WD lies in front of the RG, there is a clear rise in the line fluxes and the broadband optical light from recombination. The R-band light curve has two clear peaks near phases 1.4 and 2.4. Although our spectroscopic coverage is limited, there seems to be a maximum in $\mathrm{H} \beta$ and perhaps the other lines at phase 1.4. The peaks at phase 2.4 are obvious.

This behavior in symbiotic stars is fairly typical (e.g., Belyakina 1968; Kenyon 1982; Kenyon \& Bateson 1984; Formiggini \& Leibowitz 1990; Skopal 2008; Kenyon \& Garcia 2016). As discussed in Proga et al. $(1996,1998)$ and Skopal (2008), illumination of the RG photosphere is insufficient to generate an observable reflection effect. The RG simply does not cover enough area to intercept a significant fraction of the ionizing photons emitted by the hot WD. If the RG has an outflowing wind, however, this material is optically thick to high energy photons. The wind has a large surface area and can absorb $25 \%$ to more than $50 \%$ of the radiation emitted by the WD. Recombinations in the ionized wind produce Balmer continuum emission and strong emission lines. When the hot WD lies in front of the RG and its wind, Earth-bound observers see strong peaks in the broadband light curves and the emission line fluxes. Nearly half an orbital phase later, the illuminated hemisphere of the $\mathrm{RG}$ and its wind now lie behind the RG, which produces a strong minimum in lines and the continuum.

In a binary with a circular orbit, the maximum from illumination occurs half an orbital phase after any eclipse (e.g., Skopal 2008; Kenyon \& Garcia 2016). When the orbit is eccentric, the peak from illumination is offset from phase 0.5. Matson et al. (2016) derived a relation among the eccentricity $(e)$, the longitude of the periastron $(\omega)$ and the eclipse timings for eclipsing binaries. In terms of the orbital phase, for $i \simeq 90^{\circ}$, they obtained

$e \cos (\omega)=\frac{\pi}{2}\left(\phi_{S}-\phi_{P}-0.5\right)$,

where $\phi_{P}$ and $\phi_{S}$ are the phases for primary and secondary eclipses, respectively. If $\omega=90$ or $270^{\circ}$, when the observer views the system along the major axis of the orbit, equation 1 suggests the phase difference between successive eclipses is 0.5 for any eccentricity. But for different values of $\omega$ the situation changes. In Figure 4 we observe clearly that the primary eclipses for PU Vul, when the RG occults the WD, occur for phases 1 and 2 . The secondary eclipses take place when we observe a maximum in the light curve, at phases $\sim 1.4$ and $\sim 2.4$. As we explained previously, we observe a maximum instead of a minimum because the light comes mainly from the WD, that has a negligible size compared to the RG, and because of the reflection effect taking place where the WD illuminates the RG. Then, considering these phases in equation 1 , the offset of the illumination peak suggests the orbit in PU Vul has an eccentricity of $\approx 0.16$, when $\omega=0$ or $180^{\circ}$. This value represents a lower limit for the eccentricity, which depends on $\omega$.

\subsection{Temperatures of the hot component}

Methods for deriving the temperature of the hot component from optical emission lines rely on assumptions about the geometry of the ionized nebula. In a powerful method originally developed by Ambartsumyan (1932) and modified by Iijima (1981) to include the He I $\lambda 4471$ transition, the fluxes for He I $\lambda 4471$, He II $\lambda 4686$, and $\mathrm{H} \beta$ serve as proxies for the number of H-ionizing and He-ionizing photons. The method assumes that the nebula is optically thick to ionizing photons with $\lambda<912 \AA$; photons with $\lambda<912,504$ and $228 \AA$ ionize $\mathrm{H}^{0}, \mathrm{He}^{0}$ and $\mathrm{He}^{+}$, respectively (see Fig. 2.2 at Osterbrock 1989; Hummer \& Seaton 1964). Adopting a blackbody radiation source and electron temperatures of $10,000 \mathrm{~K}$, the temperature of the hot component is

$T_{h} \times 10^{-4}=19.38\left(\frac{2.22 F_{4686}}{4.16 F_{H_{\beta}}+9.94 F_{4471}}\right)^{1 / 2}+5.13$.

This method is valid for 70,000 $<T_{h}<200,000 \mathrm{~K}$. Aside from a central blackbody, this relation assumes that the lines form in regions with a similar geometry. If the lines are optically thin, the fluxes are independent of geometry. However, we measured that $\mathrm{H} \alpha$ is optically thick with a flux relative to $\mathrm{H} \beta$ that varies with time. The variation in this flux ratio implies a variation of the optical depth through the nebula, which may result in variations in the line fluxes with orbital phase. For PU Vul, several observations imply the assumption of line formation in regions with a similar geometry is incorrect: (i) when He II $\lambda 4686$ has a broad component, He I and H I lines do not and (ii) the variation of He II $\lambda 4686$ with orbital phase differs from the variations of the He I and $\mathrm{H}$ I lines. Still, this method gives us an idea of the variation of $T_{h}$ with time for comparison with results from UV data (Kato et al. 2011, 2012).

The upper panel of Figure 6 shows the derived temperatures (purple dots) as a function of time and phase for 
comparison with the temperatures from Kato et al. (2012, green triangles). Although the observation epochs are different, the two sets of estimates are consistent. Nevertheless, our temperatures are slightly higher near phase 1 . Considering the different methods used to derive temperatures, the agreement is reasonably good. Our results are also generally consistent with values from Tatarnikova et al. (2011), who reported an increase in temperature from $\sim 74,000 \mathrm{~K}$ in 1991 (JD 2448559) to 100,000 K in 1996 (JD 2450237). Our somewhat larger $T_{h}$ values - 100,000 K for 1991 and $\sim 125,000 \mathrm{~K}$ for 1996 - imply the same trend in the evolution of $T_{h}$ with time.

The large drop in $T_{h}$ at phase 2.4 is a result of the large illumination effect in $\mathrm{H} \beta$ and $\mathrm{He} \mathrm{I}$ 24471. During this epoch, the rise in the He II $\lambda 4686$ flux is smaller than the increases in He I $\lambda 4471$ or $\mathrm{H} \beta$; thus the derived temperature declines. It is unlikely that the actual temperature of the hot component drops during this phase. Instead, $T_{h}$ probably remains close to $200,000 \mathrm{~K}$.

The lower panel of Figure 6 shows the evolution of the U-B color, consistent with an increase of the nebular continuum that dominates optical wavelengths from 1987. The increase in $T_{h}$ correlates well with the time evolution of the U-B color. The sharp rise in $T_{h}$ at phase 0.5 coincides with a dramatic drop in $\mathrm{U}-\mathrm{B}$, from $\mathrm{U}-\mathrm{B} \approx-0.2$ to $\mathrm{U}-\mathrm{B} \approx-0.7$. From phase 0.8 to phase 2.0 , a gradual drop to $\mathrm{U}-\mathrm{B} \approx-1.2$ parallels the slow rise in $T_{h}$ from $10^{5} \mathrm{~K}$ to nearly $2 \times 10^{5} \mathrm{~K}$. After phase 2 , the $\mathrm{U}-\mathrm{B}$ color gradually reddens to $\mathrm{U}-\mathrm{B} \approx-1.1$. During this period, the apparent $T_{h}$ changes due to the illumination effect. Although illumination is also visible in the broadband photometry, there is little evolution in the broadband colors during this period.

To check our $T_{h}$ measures, we consider the method of Murset \& Nussbaumer (1994):

$$
\frac{T_{h}}{X_{\max }}=1000^{\circ} \mathrm{K} / \mathrm{eV}
$$

where $X_{\max }$ is the highest observed ionization potential. The highest ionization potentials in the observed spectra belong to $[\mathrm{Fe} \mathrm{VII}] \lambda 6087$ (ionization potential $99 \mathrm{eV}$ ) and O VI $\lambda 6830$ (ionization potential $114 \mathrm{eV}$ ), which give temperatures of $99,000 \mathrm{~K}$ and $114,000 \mathrm{~K}$, respectively. These temperatures represent lower limits, due to the lack of higher ionization potential ions observable at optical wavelengths. Similarly, the lack of a detectable [Fe X] 6374 emission line (ionization potential $233 \mathrm{eV}$ ) place a firm upper limit of $T_{h} \leq 233,000 \mathrm{~K}$. On our spectra, the first appearance of [Fe VII] and Raman-scattered O VI occurs at JD 2453525 (phase $\sim 1.8$, a little over a year after a gradual decline in U-B from -1.0 to -1.3 ), which coincides with an increase in $T_{h}$ from a little more than $100,000 \mathrm{~K}$ to close to $200,000 \mathrm{~K}$ from the $\mathrm{H}$ and He line fluxes (see also Tatarnikova et al. 2018). Thus the rise in $T_{h}$ during this epoch coincides with an increase in the ionization of the nebula.

\subsection{Luminosities of the hot component}

Estimating the luminosity of the hot component from the emission lines also relies on simple assumptions. If the hot WD radiates as a blackbody and the ionized nebula absorbs all of the $\mathrm{H}$-ionizing or $\mathrm{He}^{+}$-ionizing photons, then predic- tions from case $\mathrm{B}$ recombination theory together with the observed $\mathrm{H} \beta$ or He II $\lambda 4686$ fluxes yield the luminosity of the hot WD. If the nebula only absorbs a fraction of the ionizing photons, then the derived luminosity is a lower limit to the true luminosity. In this case, it is necessary to adopt a more detailed model to infer the luminosity of the hot WD (e.g., Proga et al. 1996, 1998). Even if the nebula is optically thick to ionizing photons, the simple blackbody + case $\mathrm{B}$ recombination model fails when the central star is a WR star (e.g., Hillier 1987). Comparisons with detailed models for WR stars then provide more accurate estimates of the luminosity (e.g., Kenyon et al. 2001). To provide continuity with previous studies and to understand the trends in the evolution from our data, we follow the standard blackbody model and note where our assumptions might be inaccurate.

Following Kenyon (1983) and Mikolajewska et al. (1997), we estimate the luminosity of the hot component from the dereddened line fluxes of $\mathrm{H} \beta$ and He II $\lambda 4686$. We assume that the hot component emits as a blackbody with $L_{h}=4 \pi r_{h}^{2} \sigma T_{h}^{4}$, where $L_{h}, r_{h}$ and $T_{h}$ are the luminosity, radius and temperature of the hot component, respectively, and $\sigma$ is the Stefan-Boltzmann constant. The observed line fluxes are related to the number of ionizing photons $(f)$, the emission coefficient $(K)$ and the recombination coefficient $(\alpha)$. For blackbody emission, $f$ is a function of $r_{h}, T_{h}$ and the energy required for ionizing $\mathrm{H}$ or $\mathrm{He}^{+}$. Combining these, for $\mathrm{H} \beta$, we derive:

$L_{h}\left(H_{\beta}\right)=\frac{4 \pi d^{2} \alpha_{H} \sigma T_{h} F\left(H_{\beta}\right)}{f_{H} p K_{\beta}}$,

where $d$ is the distance to the source, $F\left(H_{\beta}\right)$ is the observed dereddened flux in $\mathrm{H} \beta$ and $p=1.52 \times 10^{11}$ photons $\mathrm{cm}^{-2} \mathrm{~s}^{-1} \mathrm{~K}^{-3}$ is the photon emission constant. Finally, setting the values $\alpha_{H}=1.43 \times 10^{-13} \mathrm{~cm}^{3} \mathrm{~s}^{-1}$ and $K_{\beta}=0.66 \times 10^{-25} \mathrm{erg} \mathrm{cm}^{3} \mathrm{~s}^{-1}$, corresponding to a typical electron density $N_{e} \approx 10^{6} \mathrm{~cm}^{-3}$ and electron temperature $T_{e}=20,000 \mathrm{~K}$, we obtain

$L_{h}\left(H_{\beta}\right)=\frac{2.5 \times 10^{7}\left(\frac{d}{k p c}\right)^{2} T_{h} F\left(H_{\beta}\right)}{f_{H}}$,

where $L_{h}\left(H_{\beta}\right)$ is in units of solar luminosity. Adopting different values for $n_{e}$ and $T_{e}$ yield $5 \%$ to $10 \%$ differences in the numerical coefficient.

Analogously, for the luminosity from the $\mathrm{He}$ II $\lambda 4686$ flux, setting $\alpha_{\mathrm{He}^{+}}=9.08 \times 10^{-13} \mathrm{~cm}^{3} \mathrm{~s}^{-1}$ and $K_{\mathrm{He}^{+}}=7.16 \times 10^{-25} \mathrm{erg} \mathrm{cm}^{3} \mathrm{~s}^{-1}$, for a typical electron density $N_{e} \approx 10^{6} \mathrm{~cm}^{-3}$ and electron temperature $T_{e}=20,000 \mathrm{~K}$, we obtain

$L_{h}\left(H e^{+}\right)=\frac{1.5 \times 10^{7}\left(\frac{d}{k p c}\right)^{2} T_{h} F\left(H e^{+}\right)}{f_{H e^{+}}}$.

For a blackbody, $f_{H}$ and $f_{H e^{+}}$are simple functions of the temperature of the hot WD derived in the previous subsection and the energy required to ionize $H$ and $\mathrm{He}^{+}(13.6 \mathrm{eV}$ and $54.4 \mathrm{eV}$, respectively). $f_{H}$ and $f_{H e^{+}}$vary from 0 for very low $T_{h}\left(\sim 10^{3} \mathrm{~K}\right)$ to 1 for very high $T_{h}\left(\sim 10^{5} \mathrm{~K}\right)$. For an adopted distance (4.7 kpc; Kato et al. 2012) and temperature 


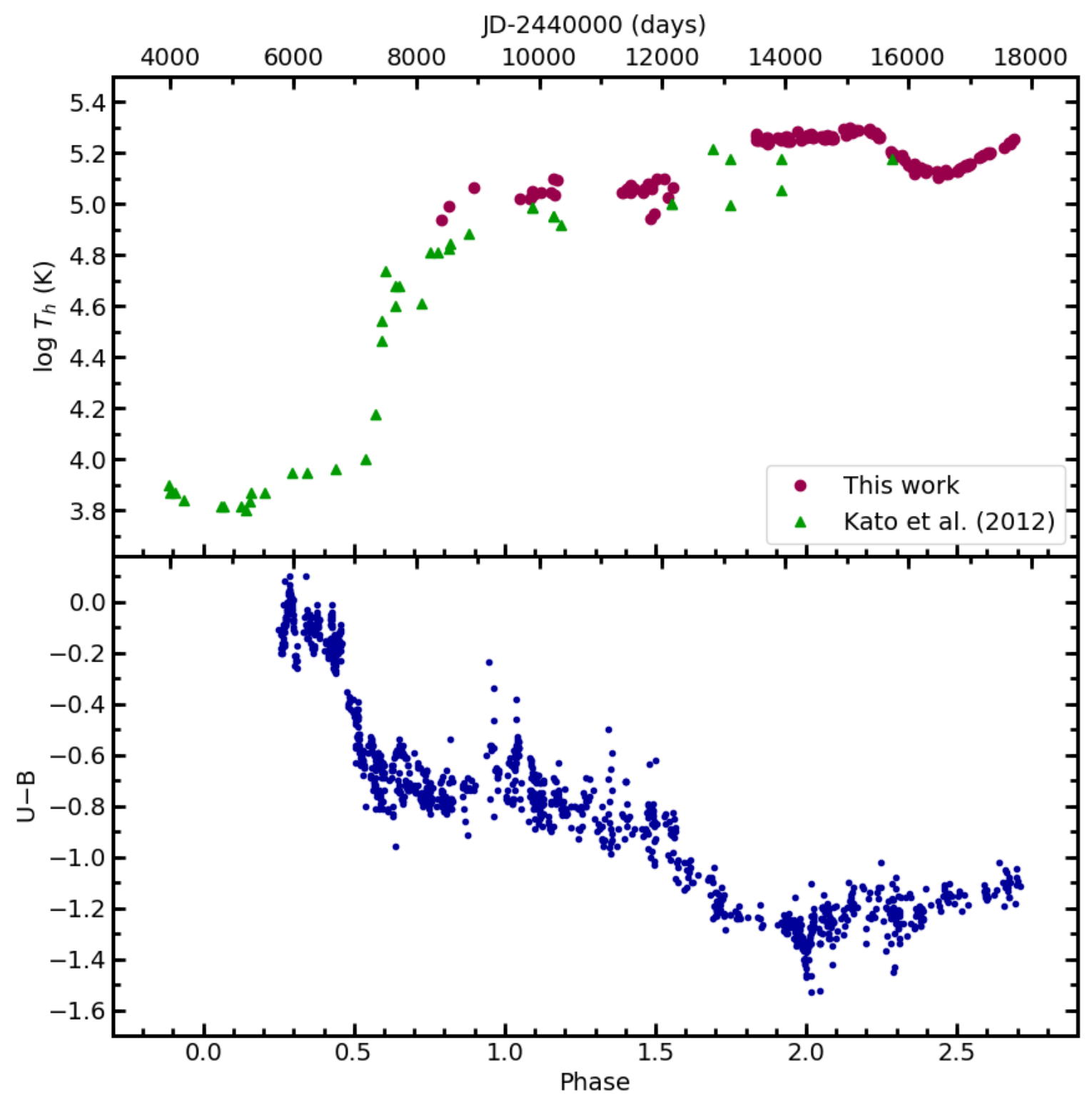

Figure 6. Evolution of the temperature of the hot component (upper panel) and the evolution of the color U-B (lower panel).

and a measured dereddened flux from $\mathrm{H} \beta$ or He II $\lambda 4686$, the luminosity follows from these two relations.

Figure 7 shows our results for the He II $\lambda 4686$ (purple dots) and the $\mathrm{H} \beta$ (yellow squares) line fluxes. The longterm evolution in $L_{h}$ is similar for the two measurements: at phases near 1.0, $L_{h}$ drops and then recovers to a slightly lower maximum level at phase 1.4. Following the maximum, $L_{h}$ falls to a distinct minimum, maintains a roughly constant value for nearly half the orbit, then rises to a clear peak at phase 2.4 , and falls once again to a low luminosity. The luminosity before and after this latest peak is basically the same: for the He II $\lambda 4686$ line the median luminosity before the peak is $1,461 \mathrm{~L}_{\odot}$, while after it the last value is $1,275 \mathrm{~L}_{\odot}$; for $\mathrm{H} \beta$, the median luminosity is $813 \mathrm{~L}_{\odot}$ before the peak and $761 \mathrm{~L}_{\odot}$ after it.

To test these results, we applied the method of Murset
\& Nussbaumer (1994) to the broadband optical photometry. In this approach, the nebula absorbs all of the high energy photons from the hot WD. Murset \& Nussbaumer (1994) adopt a density structure for the ionized nebula and use a photoionization code to predict the optical continuum from the hot WD and the surrounding nebula. Integrating over the optical passbands yields a set of bolometric corrections for the system. The luminosity of the hot WD then follows from

$\frac{L}{L \odot}=\left(\frac{d}{10 p c}\right)^{2} 10^{0.4\left(M_{b o l}^{\odot}-m_{V}+B C_{V}+A_{V}\right)}$,

where $M_{b o l}^{\odot}=4.64$ is the bolometric magnitude of the Sun, $B C_{V}$ is the bolometric correction in the $\mathrm{V}$-band and 


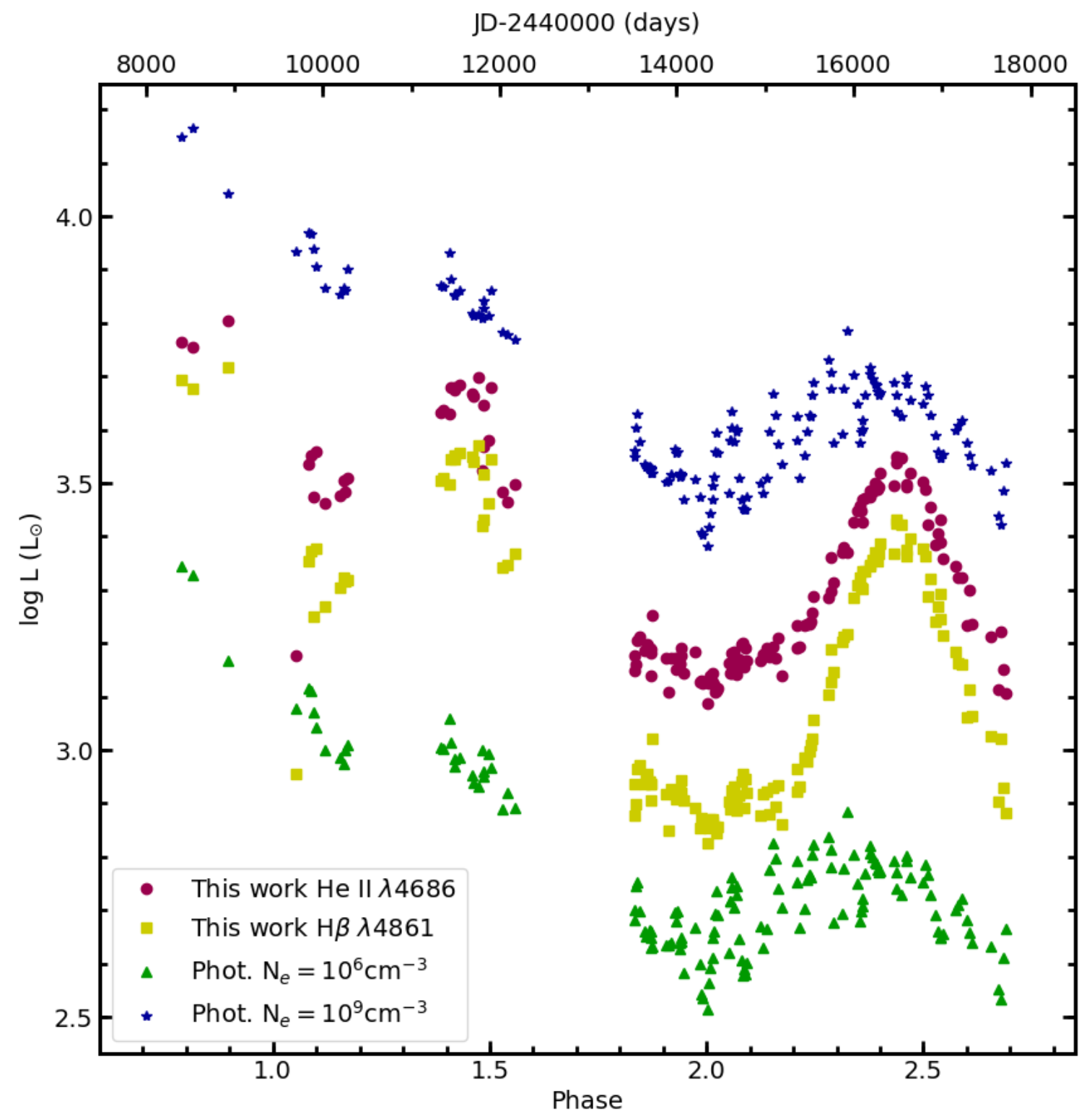

Figure 7. Luminosities from He II $\lambda 4686$ (purple dots) and $\mathrm{H} \beta$ (yellow squares) line fluxes vs time and phase, in comparison with luminosities calculated with the method of Murset \& Nussbaumer (1994, Phot.) for two different electronic densities.

$A_{V}=0.93$. Similar equations for $\mathrm{U}$ and $\mathrm{B}$ bands yield other estimates of the luminosity.

To employ the Murset \& Nussbaumer (1994) method, we must (i) estimate the contribution of the RG to the optical flux and (ii) adopt an electron density for material near the hot WD. For the $\mathrm{V}$ magnitude of the RG, a reasonable estimate is $V=13.15$, the observed brightness when the RG occults the WD and surrounding nebula near phase 2. To check this estimate, we derive an alternate V-band brightness from published IR magnitudes in Tatarnikova et al. (2011), optical colors for M5-M6 III stars in Pickles (1998), and 1 magnitude of optical extinction. The resulting magnitude, $V \approx 13.26$, is consistent with our assumption and suggests the RG contributes about half of the optical flux at phases 1.5 and 2.5 .
Murset \& Nussbaumer (1994) consider two options for the electron density. In symbiotics with short orbital periods of 2-3 years or less, the electron density is high, $\mathrm{N}_{e}=10^{9} \mathrm{~cm}^{-3}$. Systems where the RG is a Mira variable have orbital periods of decades and lower densities, $\mathrm{N}_{e}=10^{6} \mathrm{~cm}^{-3}$. Although PU Vul has some characteristics of short-period systems (e.g., a lack of warm dust near the $\mathrm{RG}$ ), its long orbital period is more characteristic of symbiotics with Mira variables. For completeness, we derive the luminosity in the high and low density limits.

Figure 7 compares the luminosities derived from the emission lines and the optical photometry. Luminosities from the Murset \& Nussbaumer (1994) method bracket the $L_{h}$ inferred from the emission lines, with the high density limit for the electron density yielding the larger $L_{h}$ estimate. The off- 
set in $L_{h}$ between the nebular $(\mathrm{H} \beta$ and $\mathrm{He}$ II) and photometric techniques is clearly a function of the adopted electron density. Apparently, an intermediate density between the two adopted values (e.g., $N_{e} \approx 3 \times 10^{7} \mathrm{~cm}^{-3}$ ) would yield a photometric $L_{h}$ more similar to the emission line result. The smaller variation of the photometric $L_{h}$ due to the illumination effect is due to the contribution of the RG. In PU Vul and other symbiotics where the hot WD has $T_{h} \approx 2 \times 10^{5} \mathrm{~K}$ and $L_{h} \gtrsim 1000 \mathrm{~L}_{\odot}$, the WD contributes no flux to the optical continuum. When the RG also contributes little or no flux, the optical continuum flux (and hence the UBV brightness) increases in step with the line flux (Aller 1984; Osterbrock 1989). In PU Vul, however, the RG emits roughly half of the $\mathrm{V}$ flux; thus the rise in the photometric $L_{h}$ at phase 2.4 is smaller than the rise in the $L_{h}$ derived from the emission lines.

Overall, our data suggest a clear drop in $L_{h}$ at orbital phases $0.8-2.8$. At the start of our observations, $L_{h} \approx 6,500 \mathrm{~L}_{\odot}$. Following the eclipse at phase $1, L_{h}$ falls to roughly $3,000 \mathrm{~L}_{\odot}$. The subsequent rise at phase 1.4 is due to the illumination effect and therefore is not a true measure of the hot component luminosity. After the illumination peak, the luminosity falls to roughly $1,000 \mathrm{~L}_{\odot}$. Except for a second rise due to illumination of the RG wind, the luminosity maintains this low level from phase 1.9 to phase 2.8 . This drop in luminosity is in agreement with Tatarnikova et al. (2011), who observed a decline by a factor 10 in the time period 1992-2008 (phases $\sim 0.8-2.1$ ).

In previous studies, Tatarnikova \& Tatarnikov (2009), Tatarnikova et al. (2011) and Kato et al. (2012) used a variety of techniques to infer $L_{h}$ for the hot WD in PU Vul (Figure 8). In their analysis of archival IUE data from 1991 to 1996, Tatarnikova \& Tatarnikov (2009) adopted a smaller distance and a larger reddening than we consider here. To make a robust comparison with our analysis, we derived $L_{h}$ from their measured continuum fluxes at $\lambda 1300$ and our adopted distance $d=4.7 \mathrm{kpc}$ and reddening $\mathrm{E}(\mathrm{B}-\mathrm{V})=0.3$. At 1991-1992 (JD 2448558-2448863), the derived $L_{h}$ is roughly a factor of two larger than our estimates, but they are comparable for the time period 1993-1996 (JD 2449161-2450343). For the same set of IUE spectra, Kato et al. (2012) calculated similar luminosities than Tatarnikova \& Tatarnikov (2009) in 1991-1992 and more than two times larger in June 1996 (JD 2450237). Aside from IUE continuum data, Kato et al. (2012) estimated $L_{h}$ from (i) integrating the spectral energy distribution, (ii) the He II $\lambda 1640$ flux, (iii) the He II $\lambda 4686$ flux, and (iv) the photometric measure of Murset \& Nussbaumer (1994). Tatarnikova et al. (2011) also derived $L_{h}$ from the He II $\lambda 4686$ flux, but adopting again different parameters than the ones we considered. We estimated luminosities from their listed line intensities, using our distance and reddening values, and the method we described above. For the time period from 2001 (JD 2452167) to 2008 (JD 2454763), the resulting $L_{h}$ is almost a factor 2 higher than our estimates, for the two lines considered. We also employed our method to estimate the luminosity from fluxes and temperature for JD 2456857, in Tatarnikova et al. (2018). For this date and for both lines, $\mathrm{H} \beta$ and He II $\lambda$ 44686, we found $L_{h}$ is higher again, but in a factor $\sim 1.3$.

Although various methods yield similar estimates for $T_{h}$ (Figure 6), there is fairly large disagreement in the de- rived luminosities. Early on in the outburst (before 1991, JD 2448000 ), the various techniques employed by Kato et al. (2012) agree and suggest a roughly constant luminosity for the hot WD. As the system continued to evolve, the IUE data suggest either (i) a factor of two drop in $L_{h}$ (Kato et al. 2012) or (ii) a factor of three drop in $L_{h}$ (Tatarnikova \& Tatarnikov 2009). Our measurements of the optical emission lines agree with the lower $L_{h}$ estimates from IUE data. Together with the optical analysis of Tatarnikova et al. (2011), our data and the optical photometry suggest another factor of four drop in $L_{h}$ from $\sim 2000$ (JD 2451600) to the present day.

Several factors play a role in the different estimates for $L_{h}$. On IUE spectra, the continuum at $\lambda 1300$ is weak and has an uncertain contribution from unresolved emission lines. The extinction correction at this wavelength is also much larger than the optical extinction. As noted earlier, the optical estimates (and UV measures based on emission lines) assume (i) the hot WD radiates as a blackbody and (ii) the nebula absorbs all H-ionizing and He-ionizing photons. Given the uncertainties, it is impressive that the various techniques yield the same general trend of a hot WD with constant luminosity for some period of time followed by a gradual decline in $L_{h}$.

\section{DISCUSSION}

After the 1979 outburst, the hot component in PU Vul initially followed the evolution expected from a WD undergoing a thermonuclear runaway (Friedjung et al. 1984; Kenyon \& Webbink 1984; Kenyon 1986b; Nussbaumer \& Vogel 1996; Kato et al. 2012; Shugarov et al. 2012). As noted by Kato et al. (2012), the WD maintained a roughly constant luminosity of $10^{4} \mathrm{~L}_{\odot}$ while the effective temperature increased from $7,000-8,000 \mathrm{~K}$ to more than $10^{5} \mathrm{~K}$. During this period, the spectrum transformed from an A-F supergiant into a WR star and then a very hot star with strong emission lines from $\mathrm{H}, \mathrm{He}$, and other species. On our optical spectra, the highest ionization features ([Fe VII] and Raman-scattered O VI) appeared in 2005 (JD 2453525) and remained strong. Although the evolution in the symbiotic nova AG Peg was much slower, it followed a similar pattern from the 1860s to the early 2000s (Kenyon et al. 1993, 2001).

Starting in 1995-2000 (JD 2449844-2451903), the derived luminosity of the hot WD began to drop (Figure 8). Despite a factor of 2-10 decline in $L_{h}$, the effective temperature of the hot WD remained roughly constant (Figure 6). This evolution runs counter to standard predictions, where the decline in $L_{h}$ occurs at roughly constant WD radius. However, the decline in $L_{h}$ is based on the assumption that the ionized nebula absorbs all of the H-ionizing and Heionizing photons from the hot WD. Our observations of $\mathrm{H}$ and He line emission are not able to test this assumption.

The broadband light curves of PU Vul (bottom panel of Figure 4) confirm some aspects of this picture. Aside from the eclipses, the initial decline of the UBVRI light curves is consistent with a hot WD evolving to higher $T_{h}$ at roughly constant luminosity. Later on, the light curves reveal two broad maxima at phases $\sim 1.4$ and 2.4 . These maxima correlate well with maxima in the emission line fluxes from $\mathrm{H} \beta$, He I $\lambda 4471$, and He II $\lambda$ 4686. The similarity between 


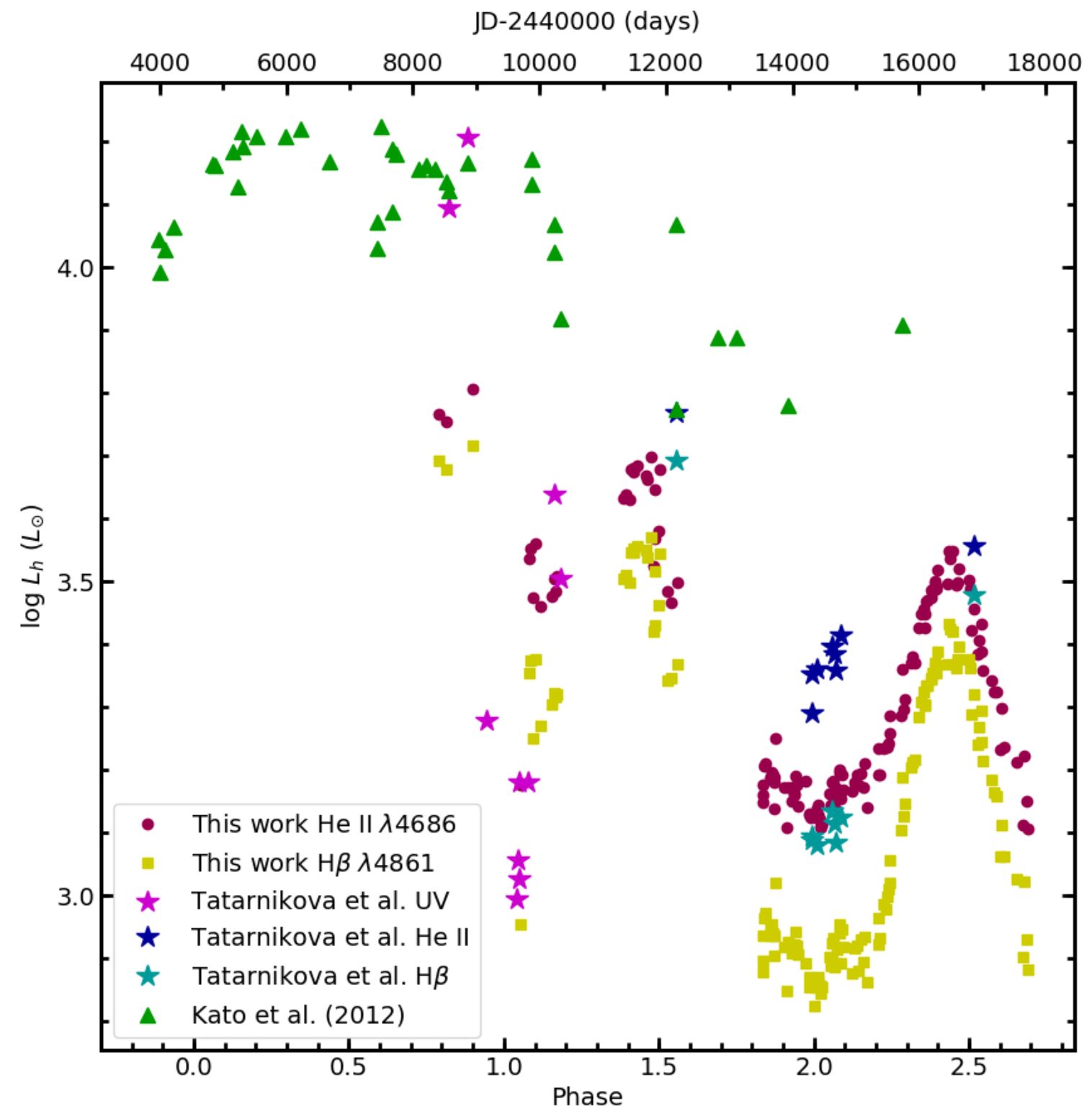

Figure 8. Luminosities from He II $\lambda 4686$ (purple dots) and $\mathrm{H} \beta$ (yellow squares) line fluxes vs time and phase, in comparison with luminosities from Tatarnikova \& Tatarnikov (2009, pink stars, UV data from JD 2448558 to 2450343), Tatarnikova et al. (2011, light and dark blue stars, optical data from JD 2452167 to 2454763), Tatarnikova et al. (2018, light and dark blue stars, optical data from JD 2456857) and Kato et al. (2012, green triangles).

the broadband light curves and the evolution of $\mathrm{H}$ and $\mathrm{He}$ emission line fluxes (3 upper panels in Figure 4) suggests that most of the $\mathrm{H}$ and He emission is generated within the ionized, outflowing wind of the RG. The slight offset of the maximum from the expected phase 1.5 (and 2.5) is consistent with an elliptical orbit, e $\geq 0.16$. Together with optical pulsations (Shugarov et al. 2012), the relative amplitudes of the illumination effect in UBVRI and the emission lines suggest the RG is responsible for roughly half of the V-band light.

Before phase 2, the behavior of the He II $\lambda 4686$ line (panel 2 of Figure 4) implies formation in the expanding WR-type wind of the hot WD (see also Tomov et al. 1991;
Andrillat \& Houziaux 1995; Kolotilov et al. 1995). The appearance of broad He II $\lambda 4686$ on our optical spectra (before phase 1.15 and between phases 1.5 and 1.6-1.8) suggest the WR wind remained fairly strong throughout the first illumination peak at phase 1.4 (when narrow emission lines dominate the spectrum). Somewhere between phases 1.6 and 1.8, the WR features fade away; the WD then gets hotter (Figure 6). The rise in the hot component temperature coincides with the appearance of the high ionization Raman-scattered O VI $\lambda 6830$ line and a significant drop in the U-B color. Several authors have noted a disappearance and re-appearance of He II $\lambda 4686$ during the second eclipse (e.g., Nussbaumer \& Vogel 1996), supporting the idea of for- 
mation close to the WD during this epoch. Although we do not have enough data around the second eclipse (phase 1) to verify the disappearance, the line is visible but weaker on 15 spectra acquired throughout the third eclipse (phase 2 ). This behavior is consistent with our contention that some He II $\lambda 4686$ emission comes from close to the hot WD, while the rest is within the ionized wind of the RG.

Aside from our picture, there are several alternative models for the time-variations in PU Vul. Kato et al. (2012) considered a model consisting of a pulsating RG (average $\mathrm{V} \approx 13.6)$, a partially ionized $\mathrm{RG}$ wind $(\mathrm{V} \approx 14$ ), a hot WD (which currently contributes little to the optical flux), and an ionized $\mathrm{WD}$ wind $(\mathrm{V} \approx 12$ during the second eclipse). Compared to our model, the RG and its wind are somewhat fainter, while the WD wind is somewhat brighter. Although this model provides a reasonable explanation for the behavior of PU Vul during the second eclipse, our analysis of the $\mathrm{H}$ and $\mathrm{He}$ lines demonstrates that emission from the ionized RG wind currently dominates emission from the WD wind. Our measurements of $\mathrm{F}(\mathrm{H} \alpha) / \mathrm{F}(\mathrm{H} \beta)$ suggests this region has a modest optical depth, $\tau_{\alpha} \approx 5-10$, in the $\mathrm{H} \alpha$ line; the optical depth in the continuum is rather small.

Tatarnikova et al. (2011) proposed that an accretion disk around the hot WD contributes an observable amount of optical and UV flux. Although Kato et al. (2012) dismissed this hypothesis due to the lack of observational evidence, disk emission is a viable explanation for the UV and optical emission of some symbiotic stars (Kenyon \& Webbink 1984). Within our set of spectra, there is little evidence for the broad emission lines expected from material orbiting the WD. Prior to phase 1.6-1.8, broad He II emission has been interpreted as emission from a WR wind instead of a disk.

Testing these proposals requires additional data analysis and better theoretical models. As we noted earlier, bolometric corrections for nebular models depend on the density of the RG wind near the hot WD (Figure 7; Murset \& Nussbaumer 1994). In principle, the relative line fluxes of various intercombination and forbidden lines provide good constraints on the electron density (e.g., Kenyon et al. 1991, 1993, and references therein). In the optical, prominent [O III], [Ne V], and [Fe VII] emission features can place good constraints on $N_{e}$ and $T_{e}$ (e.g., Nussbaumer \& Storey 1981; Nussbaumer et al. 1982; Nussbaumer \& Storey 1987). Coupled with O III], C III], and Si III] lines on IUE spectra (e.g., Nussbaumer \& Storey 1984; Nussbaumer 1986) and good reddening estimates, it should be possible to derive robust limits on the physical properties of the ionized nebulae throughout the outburst. For PU Vul, all of these lines are detected on UV and optical spectra; we defer an analysis of these features to a future study.

Placing these measurements in context requires a better understanding of the overall geometry of the RG wind. Taylor \& Seaquist (1984) analyzed different scenarios for an ionized nebula where a hot WD photoionizes the spherically symmetric wind of the RG. If the WD lies at a distance $a$ from the center of the RG, balancing recombination and ionization rates for a specified outward flow of neutral hydrogen atoms yields the shape of the surface of the resulting ionized nebula:

$X=\frac{4 \pi \mu^{2} m_{H}^{2}}{\alpha} a L_{p h}\left(\frac{\dot{M}}{v}\right)^{-2}$,

where $\mu$ is the average mass of a particle in the wind, $m_{H}$ is the mass of an hydrogen atom, $\alpha$ is the recombination coefficient, $L_{p h}=f_{H} 4 \pi R_{h}^{2} p T_{h}^{3}=\frac{f_{H} p}{\sigma}\left(\frac{L_{h}}{T_{h}}\right)$ is the luminosity of the hydrogen ionizing photons emitted per second, $\dot{M}$ is the mass loss rate and $v$ is the terminal velocity of the wind. Depending on the $X$ parameter, the system may have three different geometries:

(i) $X<\frac{1}{3}$, for an ionization bounded nebula, surrounded by the neutral part of the wind.

(ii) $\frac{1}{3}<X<\frac{\pi}{4}$, for a cone shaped ionized nebula, density limited in the outward direction, but still primarily ionization bounded.

(iii) $X>\frac{\pi}{4}$, for a density bounded nebula, with a conelike shadow zone as the neutral part of the wind and limited by the dense region near the cool star.

The emergent radio spectrum then depends on the parameter X. Originally developed for radio emission, the model is also valid for $\mathrm{H} \mathrm{I}$ Balmer lines and other emission lines. In section 4.3, we described a method to derive luminosities assuming that the nebula absorbs all of the photons from the hot component. This approach represents the usual situation in symbiotic systems and corresponds to the first case of Taylor \& Seaquist (1984). For an optically thick nebula, our results suggest a rapid drop in $L_{h}$. Alternatively, the system could have developed a density bounded nebula (e.g., $X>\frac{1}{3}$ ), where our approach underestimates the true $L_{h}$. If we set typical parameters for symbiotic stars (Seaquist et al. 1984) in equation 8 and typical parameters for PU Vul, we get:

$X=A \frac{a}{7 A U} f_{H} \frac{L_{h}}{1000 L_{\odot}} \frac{150000 K}{T_{h}}\left(\frac{10^{-7} M_{\odot} / y r}{\dot{M}} \frac{v}{10 \mathrm{~km} / \mathrm{s}}\right)^{2}$.

The chosen value for $a$ comes from using $P=13.4$ years and assuming a combined mass of $2 \mathrm{M}_{\odot}$ for the binary system (e.g. Mikołajewska 2003) in Kepler's third law: $a=$ $\left[P^{2}\left(M_{1}+M_{2}\right)\right]^{1 / 3}=7.1 \mathrm{AU}$. Then, setting $\mu=0.61$ for $\mathrm{a}$ nebula with $\mathrm{H}^{+}$and $\mathrm{He}^{++}$(see, for example, Schwarzschild 1958), $\alpha=1.43 \times 10^{-13} \mathrm{~cm}^{3} \mathrm{~s}^{-1}$ and $f_{H}=0.64-0.9$ from our calculations, we get $A \sim 11.1-15.6$. According to equation 9 the parameter $\mathrm{X}$ is basically $\mathrm{A}$ and corresponds to the third case in Taylor \& Seaquist (1984). Therefore, a better knowledge of the geometry of the nebula would allow a better determination for temperatures and luminosities of the hot component.

Although published radio observations of PU Vul are not available, high spatial VLA observations covering a broad range of wavelength could establish the geometry of the nebula (e.g., Gawryszczak et al. 2003; Angeloni et al. 2007; Vlemmings et al. 2015, and references therein). If radio data are capable of setting the $X$ parameter in the Taylor \& Seaquist (1984) model, then a combination of optical emission line and radio continuum fluxes yield better limits on $T_{h}$ and $L_{h}$. 
In addition to the Taylor \& Seaquist (1984) model, detailed models of the photoionized RG wind can also help to constrain $T_{h}$ and $L_{h}$ (e.g., Proga et al. 1996, 1998). In these calculations, optical and UV permitted, intercombination, and forbidden lines provide direct constraints on $T_{h}$ and $L_{h}$ as a function of the mass loss rate from the RG. With limits on $\dot{M}$ from radio data, it should be possible to learn whether the nebula has become density bounded or the hot WD has faded. Having a clearer picture of the evolution of the hot WD might teach us something new about thermonuclear runaways in symbiotic novae.

\section{CONCLUSIONS}

We have analyzed 32 years of optical spectroscopic observations for the symbiotic binary PU Vul. Together with the optical broadband light curve, the evolution of H I, He I, and He II emission lines reveal a clear illumination effect, where the hot WD ionizes the expanding wind of the RG. The offset of the illumination peak from mid-eclipse points to an eccentric orbit with $\mathrm{e} \geq 0.16$. In the simplest interpretation of a nebula which absorbs all ionizing photons from the hot WD, the evolution of the $\mathrm{H} \beta$ and He II $\lambda 4686$ emission lines imply an increase in effective temperature (from $T_{h} \approx 80,000 \mathrm{~K}$ to $\left.T_{h} \approx 200,000 \mathrm{~K}\right)$ and a corresponding decrease in luminosity $\left(\right.$ from $L_{h} \approx 5-10 \times 10^{3} \mathrm{~L}_{\odot}$ to $L_{h} \approx 10^{3} \mathrm{~L}_{\odot}$ ). Alternatively, the ionized nebula may have evolved from radiation bounded to density bounded. Radio observations covering a broad wavelength range, detailed analyses of optical and ultraviolet forbidden lines, and comprehensive photoionization calculations of the RG wind would test these conclusions.

\section{ACKNOWLEDGEMENTS}

We acknowledge a generous allotment of telescope time on the FLWO 1.5-m telescope and the NOAO 0.9-m telescopes. We thank various remote observers on the FLWO $1.5-\mathrm{m}$. This paper uses data products produced by the OIR Telescope Data Center, supported by the Smithsonian Astrophysical Observatory. VC is supported by the Consejo Nacional de Investigaciones Científicas y Técnicas (CONICET), Argentina. DC and SYS are supported by the Slovak Research and Development Agency under the contract No. APVV-15-0458 and by the Slovak Academy of Sciences grant VEGA No. 2/0008/17.

\section{REFERENCES}

Allen D. A., 1980, MNRAS, 192, 521

Aller L. H., ed. 1984, Physics of thermal gaseous nebulae Astrophysics and Space Science Library Vol. 112, doi:10.1007/97894-010-9639-3.

Ambartsumyan V. A., 1932, Circ. Glav. Astron. Obs., 4, 8

Andrillat Y., Houziaux L., 1995, Information Bulletin on Variable Stars, 4251

Angeloni R., Contini M., Ciroi S., Rafanelli P., 2007, A\&A, 471, 825

Argyle R. W., Liller W., Schwartz G., Whitney C. A., Kozai Y., Honda M., 1979, IAU Circ., 3348

Barnes J. V., Hayes D. S., 1982, IRS standard star manual, NOAO, Tucson
Belyakina T. S., 1968, Azh, 45, 139

Belyakina T. S., et al., 1989, A\&A, 223, 119

Chochol D., Pribulla T., Tamura S., 1998, Information Bulletin on Variable Stars, 4571

Fabricant D., Cheimets P., Caldwell N., Geary J., 1998, PASP, 110,79

Formiggini L., Leibowitz E. M., 1990, A\&A, 227, 121

Friedjung M., Ferrari-Toniolo M., Persi P., Altamore A., Cassatella A., Viotti R., 1984, in Mead J. M., Chapman R. D., Kondo Y., eds, NASA Conference Publication Vol. 2349, NASA Conference Publication.

Garnavich P. M., 1996, Journal of the American Association of Variable Star Observers (JAAVSO), 24, 81

Gawryszczak A. J., Mikołajewska J., Różyczka M., 2003, A\&A, 398, 159

Gochermann J., 1991, A\&A, 250, 361

Hayes D. S., Latham D. W., 1975, ApJ, 197, 593

Hillier D. J., 1987, ApJS, 63, 965

Hummer D. G., Seaton M. J., 1964, MNRAS, 127, 217

Iben Jr. I., 1982, ApJ, 259, 244

Iben Jr. I., Tutukov A. V., 1996, ApJS, 105, 145

Iijima T., 1981, in Carling E. B., Kopal Z., eds, Photometric and Spectroscopic Binary Systems. p. 517

Iijima T., 1989, A\&A, 215, 57

Ivison R. J., Bode M. F., Roberts J. A., Meaburn J., Davis R. J., Nelson R. F., Spencer R. E., 1991, MNRAS, 249, 374

Kanamitsu O., 1991, PASJ, 43, 225

Kanamitsu O., Yamashita Y., Norimoto Y., Watanabe E., Yutani M., 1991, PASJ, 43, 523

Kato M., 2002, in Gänsicke B. T., Beuermann K., Reinsch K., eds, Astronomical Society of the Pacific Conference Series Vol. 261, The Physics of Cataclysmic Variables and Related Objects. p. 595 (arXiv:astro-ph/0110312)

Kato M., Hachisu I., Cassatella A., González-Riestra R., 2011, ApJ, 727, 72

Kato M., Mikołajewska J., Hachisu I., 2012, ApJ, 750, 5

Kenyon S. J., 1982, PASP, 94, 165

Kenyon S. J., 1983, PhD thesis, Illinois Univ., UrbanaChampaign.

Kenyon S. J., 1986a, The symbiotic stars

Kenyon S. J., 1986b, AJ, 91, 563

Kenyon S. J., Bateson F. M., 1984, PASP, 96, 321

Kenyon S. J., Garcia M. R., 2016, AJ, 152, 1

Kenyon S. J., Truran J. W., 1983, ApJ, 273, 280

Kenyon S. J., Webbink R. F., 1984, ApJ, 279, 252

Kenyon S. J., Oliversen N. A., Mikolajewska J., Mikolajewski M., Stencel R. E., Garcia M. R., Anderson C. M., 1991, AJ, 101, 637

Kenyon S. J., Mikolajewska J., Mikolajewski M., Polidan R. S., Slovak M. H., 1993, AJ, 106, 1573

Kenyon S. J., Proga D., Keyes C. D., 2001, AJ, 122, 349

Klein A., Bruch A., Luthardt R., 1994, A\&AS, 104, 99

Kolotilov E. A., Munari U., Yudin B. F., 1995, MNRAS, 275, 185

Kozai Y., Kuwano Y., Mattei J., Annal R., 1979, IAU Circ., 3344

Liller M. H., Liller W., 1979, AJ, 84, 1357

Massey P., Strobel K., Barnes J. V., Anderson E., 1988, ApJ, 328, 315

Matson R. A., Gies D. R., Guo Z., Orosz J. A., 2016, AJ, 151, 139

Mikołajewska J., 2003, in Corradi R. L. M., Mikolajewska J., Mahoney T. J., eds, Astronomical Society of the Pacific Conference Series Vol. 303, Symbiotic Stars Probing Stellar Evolution. p. 9 (arXiv:astro-ph/0210489)

Mikolajewska J., Kenyon S. J., 1992, MNRAS, 256, 177

Mikolajewska J., Acker A., Stenholm B., 1997, A\&A, 327, 191

Murset U., Nussbaumer H., 1994, A\&A, 282, 586

Nussbaumer H., 1986, A\&A, 155, 205

Nussbaumer H., Storey P. J., 1981, A\&A, 99, 177 
Nussbaumer H., Storey P. J., 1984, A\&AS, 56, 293

Nussbaumer H., Storey P. J., 1987, A\&AS, 69, 123

Nussbaumer H., Vogel M., 1987, A\&A, 182, 51

Nussbaumer H., Vogel M., 1996, A\&A, 307, 470

Nussbaumer H., Storey P. J., Storey P. J., 1982, A\&A, 113, 21

Osterbrock D. E., 1989, Astrophysics of gaseous nebulae and active galactic nuclei

Pickles A. J., 1998, PASP, 110, 863

Press W. H., Teukolsky S. A., Vetterling W. T., Flannery B. P., 1992, Numerical recipes in FORTRAN. The art of scientific computing

Proga D., Kenyon S. J., Raymond J. C., Mikolajewska J., 1996, ApJ, 471, 930

Proga D., Kenyon S. J., Raymond J. C., 1998, ApJ, 501, 339

Savage B. D., Mathis J. S., 1979, ARA\&A, 17, 73

Schwarzschild M., 1958, Structure and evolution of the stars.

Seaquist E. R., Taylor A. R., Button S., 1984, ApJ, 284, 202

Shugarov S., Chochol D., Kolotilov E., 2012, Baltic Astronomy, 21,150

Sion E. M., Shore S. N., Ready C. J., Scheible M. P., 1993, AJ, 106,2118

Skopal A., 2008, Journal of the American Association of Variable Star Observers (JAAVSO), 36, 9

Starrfield S., Truran J. W., Sparks W. M., Kutter G. S., 1972, ApJ, 176, 169

Tatarnikova A. A., Tatarnikov A. M., 2009, Astronomy Reports, 53,1020

Tatarnikova A. A., Tatarnikov A. M., Esipov V. F., Tarasova T. N., Shenavrin V. I., Kolotilov E. A., Nadzhip A. E., 2011, Astronomy Reports, 55, 896

Tatarnikova A., et al., 2018, Research in Astronomy and Astrophysics, submitted

Taylor A. R., Seaquist E. R., 1984, ApJ, 286, 263

Tomov T., Zamanov R., Iliev L., Mikolajewski M., Georgiev L., 1991, MNRAS, 252, 31P

Vlemmings W. H. T., Ramstedt S., O'Gorman E., Humphreys E. M. L., Wittkowski M., Baudry A., Karovska M., 2015, A\&A, 577, L4

Vogel M., Nussbaumer H., 1992, A\&A, 259, 525

Yamashita Y., Maehara H., Norimoto Y., 1982, PASJ, 34, 269

Yaron O., Prialnik D., Shara M. M., Kovetz A., 2005, ApJ, 623, 398

This paper has been typeset from a $\mathrm{T}_{\mathrm{E}} \mathrm{X} / \mathrm{LAT}_{\mathrm{E}} \mathrm{X}$ file prepared by the author. 